\title{
CT Protocols for Attenuation Correction and Segmentation in 99mTc-MAA SPECT/CT for 90Y Radioembolization Treatment Planning - a Simulation Study
}

\section{Zhonglin Lu}

\author{
University of Macau
}

Greta S. P. Mok ( $\sim$ gretamok@um.edu.mo)

University of Macau https://orcid.org/0000-0002-2962-2087

Original research

Keywords: $90 \mathrm{Y}$ radioembolization, 99mTc-macroaggregated albumin, SPECT/CT, attenuation correction, segmentation, lung shunt fraction, tumor-to-normal liver ratio, injected activity, partition model

Posted Date: May 28th, 2020

DOl: https://doi.org/10.21203/rs.3.rs-31563/v1

License: (c) (1) This work is licensed under a Creative Commons Attribution 4.0 International License.

Read Full License 
3 Planning - a Simulation Study

4

5 Zhonglin $\mathrm{Lu}^{1}$ and Greta S. P. Mok ${ }^{1,2 *}$

6

7 Macau, Avenida da Universidade, Taipa, Macau SAR, China

*Correspondence: gretamok@um.edu.mo.

CT Protocols for Attenuation Correction and Segmentation in ${ }^{99}{ }^{9}$ Tc-MAA SPECT/CT for ${ }^{90}$ Y Radioembolization Treatment

${ }^{1}$ Biomedical Imaging Laboratory (BIG), Department of Electrical and Computer Engineering, Faculty of Science and Technology $\&^{2}$ Center for Cognitive and Brain Sciences, Institute of Collaborative Innovation, University of 


\section{Abstract}

Background: Conventional ${ }^{99 \mathrm{~m}} \mathrm{Tc}-$ macroaggregated albumin $\left({ }^{99 \mathrm{~m}} \mathrm{Tc}-\mathrm{MAA}\right)$ planar scintigraphy overestimates lung shunt fraction (LSF) as compared to SPECT/CT in ${ }^{90} \mathrm{Y}$ radioembolization treatment planning. However, the respiratory motion artifact due to the temporal mismatch between static SPECT and helical CT (HCT) may compromise the SPECT quantitation accuracy by incorrect attenuation correction (AC) and volume-of-interest segmentation. The goal of this study is to systematically assess different AC and segmentation protocols for LSF, tumor-tonormal liver (TNR), organ absorbed dose and injected activity (IA) estimation in ${ }^{99 \mathrm{~m}}$ Tc-MAA SPECT/CT.

Methods: The 4D XCAT phantom was used to simulate 10 patient anatomies with ${ }^{99 \mathrm{~m}} \mathrm{Tc}-\mathrm{MAA}$ distribution based on the clinical data, each with LSF of 5\%,10\%, 15\% and 20\%, axial respiratory motion of $2 \mathrm{~cm}$, different TNR and tumor size. An analytical projector for low energy high resolution parallel-hole collimator was used to simulate realistic noisy planar acquisitions, and 128 projections over $360^{\circ}$ for SPECT, both modeling attenuation, scatter and geometric collimator-detector-response. Five attenuation maps, i.e., (i) HCT at end-inspiration (HCT-IN), (ii) $\mathrm{HCT}$ at mid-respiration (HCT-MID), (iii) HCT at end-expiration (HCT-EX), (iv) cine averaged CT (CACT) and (v) interpolated average CT (IACT) were applied for SPECT AC and segmentation in LSF, dosimetric and IA evaluation. Mid-respiratory phases were also extracted from CACT/IACT for VOI segmentation while CACT/IACT were used for AC, i.e., hybrid CT protocols.

Results: For LSF estimation, SPECT/CACT has the least absolute errors. Planar significantly overestimates LSF and lung absorbed dose compared to SPECT especially for LSF of 5\%. SPECT-based is better than CT-based segmentation in TNR estimation. There is no statistically 
49 significant difference for different CT protocols for TNR, normal liver and tumor absorbed dose 50 estimation. Hybrid CTs and HCT-MID performed the best for IA especially for higher LSF.

51 Conclusions: ${ }^{99 \mathrm{~m}} \mathrm{Tc}-\mathrm{MAA}$ SPECT/CT with an appropriate choice of CT protocol for AC and 52 segmentation is superior to planar in LSF and lung absorbed dose estimation. The 4D CT 53 protocols are recommended for $\mathrm{AC}$ and segmentation to alleviate respiratory artifacts and 54 improve quantitation accuracy in ${ }^{90} \mathrm{Y}$ radioembolization treatment planning. HCT-EX would also 55 be a recommended choice if 4D CT is not available.

56

57 Keywords: ${ }^{90} \mathrm{Y}$ radioembolization; ${ }^{99 \mathrm{~m}} \mathrm{Tc}-$ macroaggregated albumin; SPECT/CT; attenuation 58 correction; segmentation; lung shunt fraction; tumor-to-normal liver ratio; injected activity; 59 partition model. 
${ }^{90} \mathrm{Y}$ radioembolization, also known as selective internal radiation therapy (SIRT) is increasingly adopted as an efficient treatment for patients with inoperable hepatocellular carcinoma (HCC) and liver metastasis, using microspheres loaded with radioactive isotope ${ }^{90} \mathrm{Y}$ whose primary $\beta^{-}$ emission energy is $2.28 \mathrm{MeV}$ [1]. Two types of commercial ${ }^{90} \mathrm{Y}$ microspheres are currently

72 available: resin microspheres (SIR-Sphere®, Sirtex, Medical, Sydney, Australia) and glass microspheres (TheraSphere ${ }^{\circledR}$, BTG, Boston Scientific, Massachusetts, United States). These microspheres are injected through a catheter connecting to the hepatic artery which mainly supplies the tumor while portal vein feeds the normal liver parenchyma, resulting in high radiation dose to tumor when sparing normal liver [2]. Complications of ${ }^{90} \mathrm{Y}$ radioembolization are mainly radiation-induced liver disease, radiation pneumonitis, gastrointestinal ulceration and diarrhea [3]. Thus, prior to ${ }^{90} \mathrm{Y}$ microspheres treatment, appropriate imaging evaluation should be performed to determine parameters related to complications such as lung shunting fraction (LSF), tumor-to-normal liver ratio (TNR) and injected activity (IA) based on the partition model [4]. Radionuclide imaging is a prevailing method to investigate these parameters, using ${ }^{99 \mathrm{~m}} \mathrm{Tc}-$ macroaggregated albumin $\left({ }^{99 \mathrm{~m}} \mathrm{Tc}-\mathrm{MAA}\right)$ as a surrogate for ${ }^{90} \mathrm{Y}$ microsphere [5].

Conventional ${ }^{99 m}$ Tc-MAA planar imaging is limited in lungs and liver delineation due to organs overlap and lack of anatomical references. Additionally, planar images suffer from poor image quality due to the limited compensations available. Therefore, previous studies reported that the LSF was over estimated using planar scintigraphy as compared to quantitative ${ }^{99 \mathrm{~m}} \mathrm{Tc}-$ MAA SPECT/CT [6-8]. In addition, TNR, tumor and normal liver volume can be measured on SPECT/CT or contrast CT for ${ }^{90} \mathrm{Y}$ treatment planning [5].

Nonetheless, the mismatch between the sequential SPECT and conventional helical CT (HCT) 
acquisitions leads to the respiratory artifacts on SPECT reconstructed images especially near the diaphragmatic region of the lower lungs and upper liver $[9,10]$, mainly due to the improper attenuation correction (AC) $[11,12]$. That would potentially degrade the ${ }^{99 \mathrm{~m}}$ Tc-MAA SPECT/CT quantitative accuracy, affecting the LSF and TNR calculation. The use of average CT, including cine average CT (CACT) and our previously proposed interpolated average CT (IACT), has been proven to be an effective AC method to reduce the respiratory artifacts and improve the quantification for thoracic SPECT [11] and PET [13-15]. Besides, segmentation of lungs, liver and tumors also highly affects the dosimetric accuracy for ${ }^{99 \mathrm{~m}}$ Tc-MAA SPECT/CT [16]. To the best of our knowledge, the optimal CT protocol has not yet been determined for ${ }^{99 \mathrm{~m}}$ Tc-MAA SPECT/CT. This study aims to evaluate the use of different CT protocols, including HCTs acquired at end-inspiration (HCT-IN), end-expiration (HCT-EX), mid-respiration (HCT-MID), CACT and IACT as AC maps and segmentation references for ${ }^{99 \mathrm{~m}}$ Tc-MAA SPECT/CT based on simulations with known truth. The LSF and lung absorbed dose measured from the planar scintigraphy were also shown references.

\section{Materials and Methods}

\section{Phantom population}

The 4D digital extended cardiac torso (XCAT) phantom [17] was employed in this study to model 10 different patient anatomies varying in gender, body size and heart size [18] as well as TNR (4 to 11.4), spherical tumor size (2 $\mathrm{cm}$ to $8.7 \mathrm{~cm}$ in diameter) and location (Table 1) based on the clinical data with ${ }^{99 m}$ Tc-MAA distribution (Table 2) [19]. In addition, each patient anatomy was modeled with four different LSFs, i.e., 5\%, 10\%, 15\% and 20\% (Fig. 1). Axial 
112 respiratory motion of $2 \mathrm{~cm}$ and anterior-posterior expansion of $1.2 \mathrm{~cm}$ with a period of $4.8 \mathrm{~s}$

113 divided into 24 frames was modeled for the respiratory cycle. The average activity map of 24

114 frames and the corresponding averaged attenuation map at $140 \mathrm{keV}$ were generated. For the

115 purpose of modeling the continuous nature of the activity distribution, XCAT phantoms with a

116 voxel size of $0.221 \mathrm{~cm}$ were collapsed to a bin size of $0.442 \mathrm{~cm}$.

117

Table 1. Anatomy of XCAT phantoms

\begin{tabular}{|c|c|c|c|c|}
\hline & Lung volume (ml) & $\begin{array}{c}\text { Normal liver } \\
\text { volume (ml) }\end{array}$ & $\begin{array}{c}\text { Tumor } \\
\text { volume (ml) }\end{array}$ & TNR \\
\hline Phantom \#1 & 3403 & 1855 & 17 & 11.4 \\
\hline Phantom \#2 & 4917 & 1996 & 346 & 6 \\
\hline Phantom \#3, \#4 & 2564 & 1084 & 48 & 8.2 \\
\hline Phantom \#5 & 3622 & 1624 & 6 & 7 \\
\hline Phantom \#6 & 3403 & 1855 & 125 & 11.4 \\
\hline Phantom \#7 & 3403 & 1747 & 125 & 5.2 \\
\hline Phantom \#8 & 2446 & 929 & 74 & 4 \\
\hline Phantom \#9 & 3530 & 1555 & $66 / 23$ & 9 \\
\hline Phantom \#10 & 4876 & 2269 & & 7 \\
\hline
\end{tabular}

"Phantom \#3 and Phantom \#4 have different tumor locations. 
Table 2. ${ }^{99 \mathrm{~m}}$ Tc-MAA distribution of XCAT phantoms

\begin{tabular}{|c|c|c|c|c|c|c|}
\hline Unit: & \multicolumn{4}{|c|}{ Lungs } & \multirow{2}{*}{$\begin{array}{c}\text { Normal } \\
\text { liver }\end{array}$} & \multirow[t]{2}{*}{ Tumor } \\
\hline Counts/voxel & $5 \% \mathrm{LSF}$ & $10 \% \mathrm{LSF}$ & $15 \% \mathrm{LSF}$ & $20 \% \mathrm{LSF}$ & & \\
\hline Phantom \#1 & 22 & 47 & 75 & 106 & \multirow{9}{*}{700} & 8000 \\
\hline Phantom \#2 & 30 & 64 & 102 & 145 & & 4200 \\
\hline Phantom \#3, \#4 & 21 & 45 & 71 & 101 & & 5740 \\
\hline Phantom \#5 & 17 & 36 & 57 & 80 & & 4900 \\
\hline Phantom \#6 & 21 & 45 & 72 & 102 & & 4900 \\
\hline Phantom \#7 & 34 & 73 & 115 & 164 & & 8000 \\
\hline Phantom \#8 & 24 & 50 & 80 & 113 & & 3640 \\
\hline Phantom \#9 & 19 & 41 & 65 & 92 & & 2800 \\
\hline Phantom \#10 & 23 & 49 & 78 & 110 & & 6300 \\
\hline
\end{tabular}

130 FIG .1. Phantom \#6 with LSF of (a) 5\%, (b) 10\%, (c) $15 \%$ and (d) $20 \%$.

\section{Simulation study}

We simulated a standard clinical dual head SPECT/CT system with a crystal thickness of 2.54 $\mathrm{cm}$ mounted with a low energy high resolution (LEHR) parallel-hole collimator using an analytical projector [20]. For the planar protocol, anterior and posterior planar images were generated in a supine position for $5 \mathrm{~min} / \mathrm{view}$, covering both lungs and the liver. For the SPECT/CT protocol, 128 projections were generated over $360^{\circ}$ with 30 s/view, using $20 \%$ energy window centered at the photopeak of $140 \mathrm{keV}$. Both protocols modeled attenuation, 
scatter and collimator-detector response (CDR). The scatter modeling (for SPECT and planar)

140

141

142

143

144

145

146

147

148

149

150

151

152

153

154

155

156

157

158

159

160 and correction (for SPECT only) were achieved by the effective source scatter estimation method [21]. The Poisson noise was modelled in the SPECT projections and planar images (Fig. 2) based on the normalized standard deviation (NSD) of a relatively uniform region-of-interest (ROI) drawn in the liver region of the clinical SPECT projection data and planar data, respectively. For the clinical count level reference, the injected ${ }^{99 \mathrm{~m}}$ Tc-MAA activity was $111 \mathrm{MBq}$. The one bed position (axial field of view of $40 \mathrm{~cm}$ ) SPECT acquisition time was 25 s/projection for 60 projections, while the planar acquisition was also with one bed position (covering chest to liver) and $1000 \mathrm{kcts}$ were collected. There was no AC or scatter correction (SC) performed in planar images [22]. The average attenuation maps as well as the attenuation maps of the XCAT phantoms in end-inspiration, middle-respiration and end-expiration phases were used to generate CT projections by an analytical projector and then added with Gaussian noise whose level was determined based on the clinical CT data [23]. The noisy projections were then reconstructed using a filtered back-projection algorithm to obtain corresponding noisy CACT, HCT-IN, HCTMID and HCT-EX (Fig. 3a-d). The HCT-IN and HCT-EX were applied to generate IACT (Fig. 3e) via image registration and interpolation based on the standard XCAT respiratory curve [23, 24]. Additionally, another set of contrast HCT-IN, HCT-MID and HCT-EX with enhanced tumor intensity was modeled.

Ordered subset expectation maximization (OS-EM) with 4 iterations and 16 subsets, i.e., a total of 64 updates, was used for image reconstruction with AC, SC and CDR modelling during the reconstruction. Different $\mathrm{CT}$ attenuation maps were used for $\mathrm{AC}$ in reconstruction respectively. 


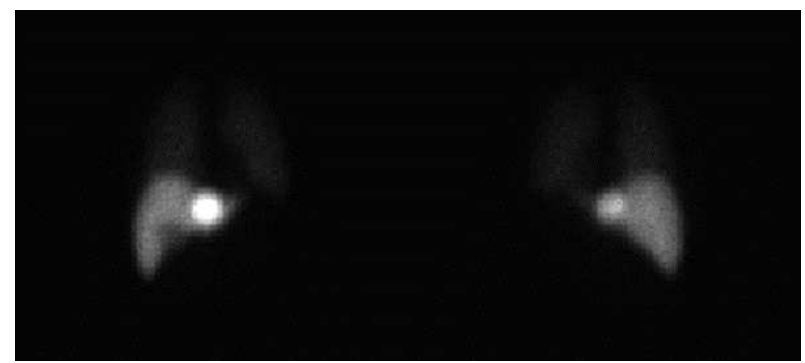

FIG. 2. Noisy anterior (left) and posterior (right) planar images of Phantom \#3.
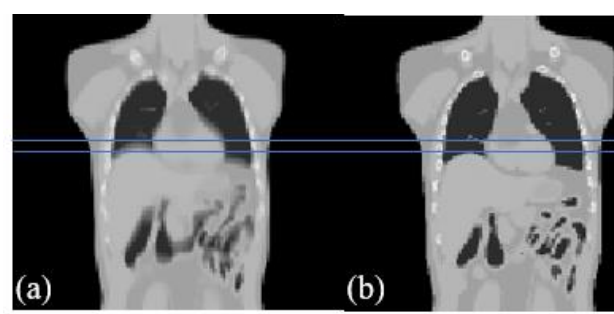

(c)

(d)

(e)

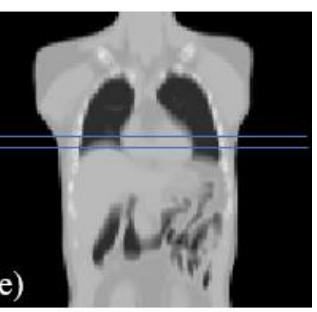

165 FIG. 3. (a) CACT, (b) HCT-IN, (c) HCT-MID, (d) HCT-EX and (e) IACT of Phantom \#1. Blue

166 lines represent motion range of $2 \mathrm{~cm}$.

\section{Data analysis}

\section{LSF estimation}

170 For planar images, ROIs of the lungs and livers were manually delineated on anterior and

171 posterior views. Left and right lungs were segmented out together and there was a gap of about 1

$172 \mathrm{~cm}$ between the liver and lung interface (Fig. 4) [8]. In this study, the geometric mean (GM) was

173 measured for the lungs and liver using the following equation.

$$
\mathrm{GM}=\sqrt{C_{A} \times C_{P}}
$$

175 where $C_{A}$ and $C_{P}$ are the total counts in the ROIs on anterior and posterior views, respectively. The LSF was then computed using Eq. 2.

$$
\mathrm{LSF}=\frac{G M_{\text {lung }}}{G M_{\text {lung }}+G M_{\text {liver }}}
$$


where $G M_{\text {lung }}$ and $G M_{\text {liver }}$ are the geometric mean of the lungs and liver, respectively.
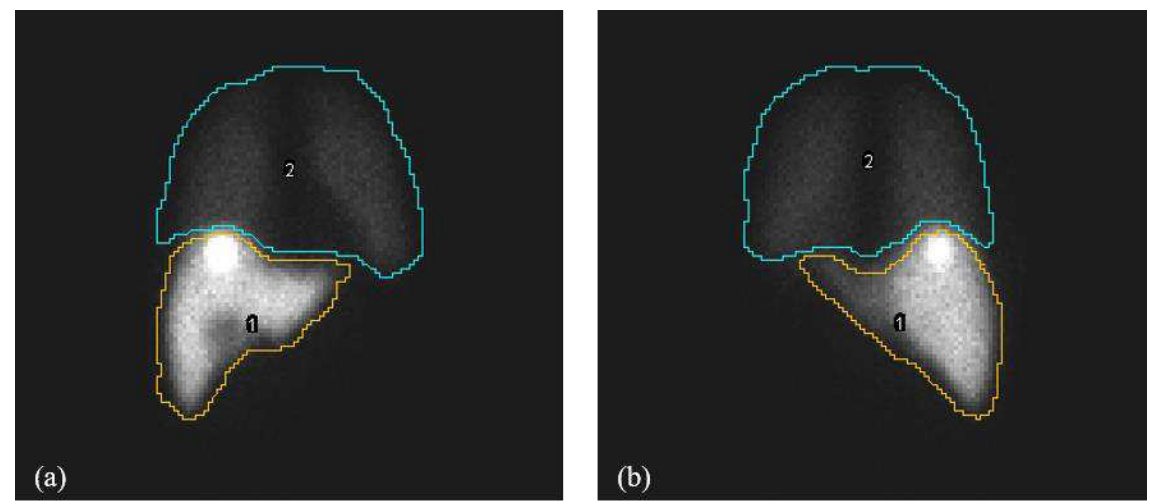

FIG. 4. Liver (orange ROI) and lung segmentation (blue ROI) of planar images on (a) anterior and (b) posterior view.

For quantitative SPECT/CT images, an open-source semi-automatic software ITK-SNAP was used for segmentation [25]. Lung volumes-of-interest (VOIs) were automatically segmented on CT images by setting an upper threshold of -200 Hounsfield unit (HU), with manual removal of the main trachea. To reduce the impact of respiratory motion spilling liver activity into the lungs, an axial range of $2.21 \mathrm{~cm}$ of the basal lung VOI was excluded as shown in Fig. 5 (a) [6]. The total lung counts can be estimated by the product of the lung mean count density (counts/voxel) of the lung VOI after $2.21 \mathrm{~cm}$ exclusion and the original CT lung volume determined from each CT protocol [7]. For liver segmentation, it can be done on the SPECT or CT images. Thus, liver VOIs were segmented on SPECT images directly by setting a lower threshold of $2.5 \%$ of the maximum count value (SPECT-based liver segmentation method) (Fig. 5b) [8] or contoured on different CT images (Fig. 5c) and then mapped to the corresponding SPECT images (CT-based liver segmentation method). The LSF on SPECT was calculated using Eq. 3.

$$
\mathrm{LSF}=\frac{\text { lung counts }}{\text { liver counts }+ \text { lung counts }}
$$



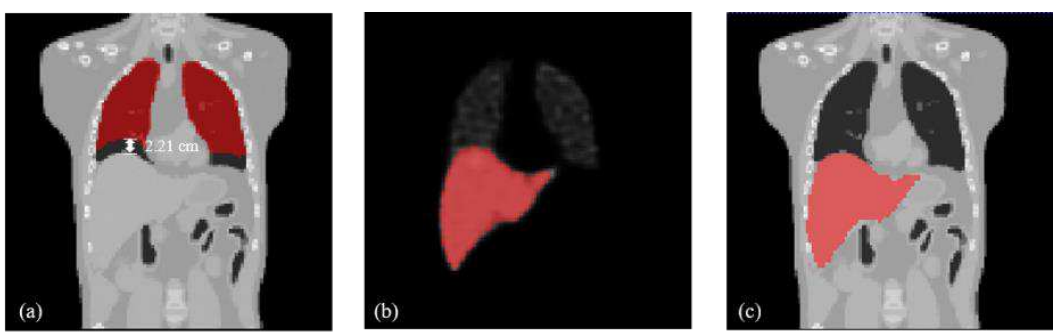

198

199

200

201

FIG. 5. (a) Lung segmentation with $2.21 \mathrm{~cm}$ axial exclusion on HCT-MID.(b) Liver segmentation on SPECT image and on (c) HCT-MID for Phantom \#1.

The SPECT-based or CT-based liver segmentation methods with lower mean absolute error would then be applied to estimate LSF for different CT protocols.

\section{TNR estimation}

Only ${ }^{99 m}$ Tc-MAA SPECT/CT images were used for TNR calculation. SPECT reconstructed images using CACT for AC were first employed to investigate the best segmentation protocols of the normal liver (NL) and tumor VOIs for TNR estimation. The tumor and NL VOIs were first contoured on contrast HCT-IN, HCT-MID (Fig. 6a, b) and HCT-EX images respectively. The tumor and NL VOIs would then be mapped to the SPECT images (Fig. 6c, d). The NL and tumor VOIs can also be segmented from intensity thresholding on SPECT images. The whole liver was segmented by setting a lower threshold of $2.5 \%$ of maximum intensity while the tumor was segmented by setting a lower threshold of $40 \%$ (Fig. 7a) and 50\% (Fig. 7c) of maximum intensity on SPECT images, defined as SPECT(40\%) and SPECT(50\%), respectively. The NL VOIs (Fig. 7b, d) were obtained by subtracting corresponding tumor VOIs from the whole liver VOI. The third segmentation protocol was to delineate the NL and tumor VOIs by combining information from SPECT and contrast HCT-EX images, i.e., SPECT+contrast HCT-EX protocol. 
217 The NL (Fig. 8b) and tumor (Fig. 8a) VOIs were determined on SPECT images by setting a

218 threshold such that the volume of VOIs equaled to that of corresponding VOIs from the contrast

219 HCT-EX image [26]. The tumor and NL VOIs obtained from these methods can then be used to

220 calculate TNR using Eq. 4 [4, 27].

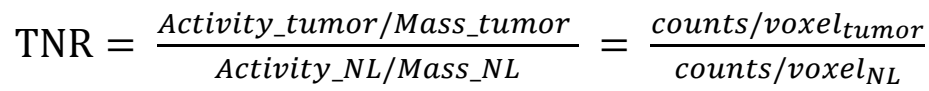

222 where counts/voxel is defined as mean count density.
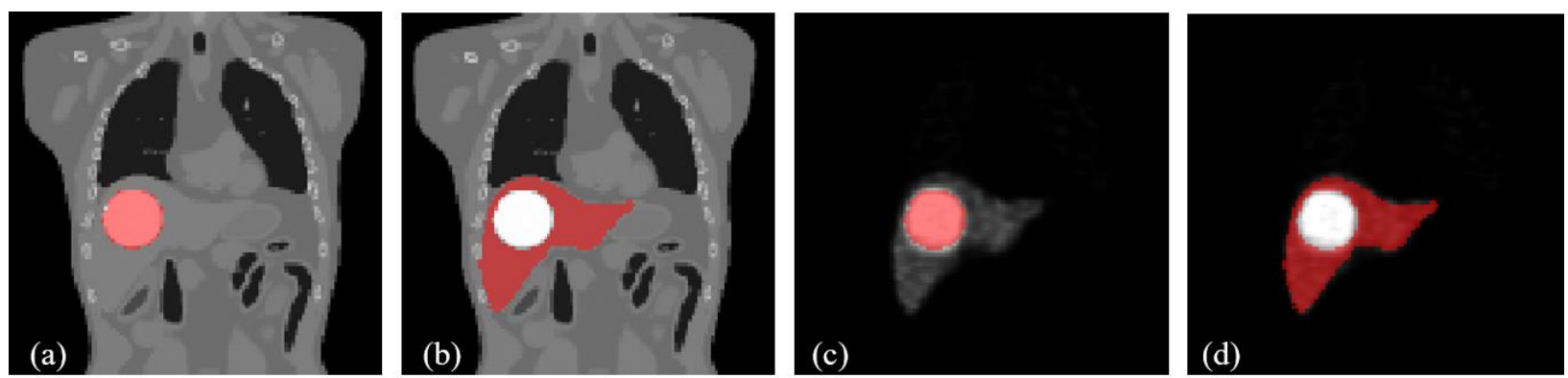

FIG. 6. (a) Tumor and (b) NL segmentation on contrast HCT-MID. Corresponding VOIs were then mapped to segment the (c) tumor and (d) NL on SPECT images for Phantom \#2.
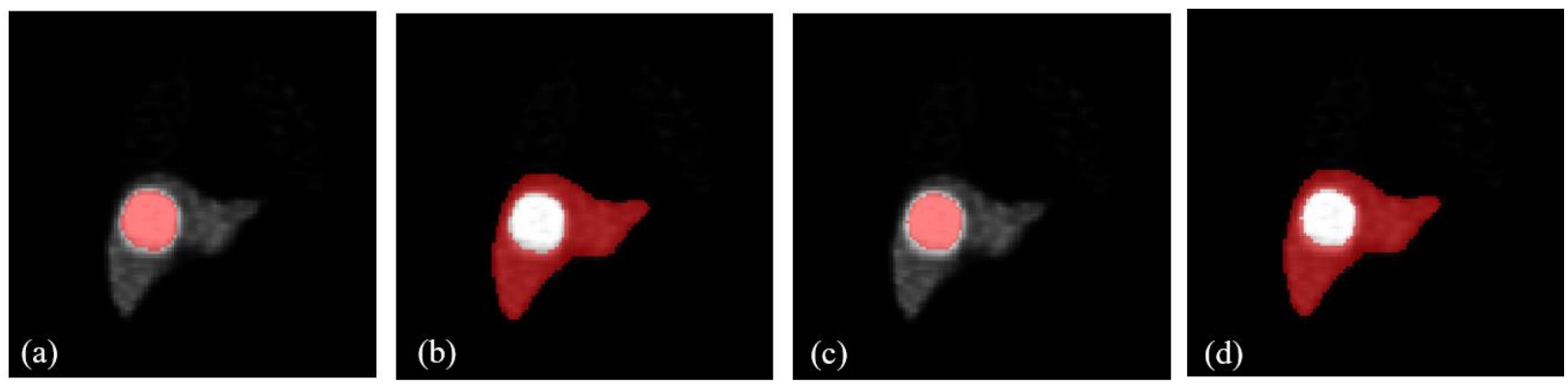

FIG. 7. (a) Tumor segmented from $40 \%$ of maximum intensity and (b) corresponding NL VOI.

(c) Tumor segmented from $50 \%$ of maximum intensity and (d) corresponding NL VOI on 

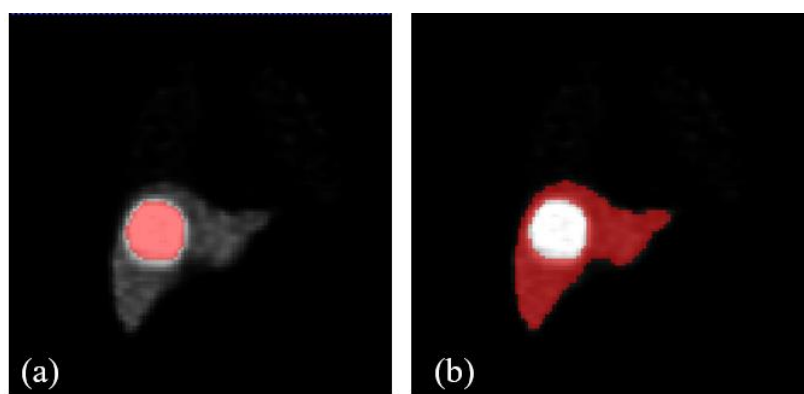

FIG. 8. (a) Tumor and (b) NL VOIs on SPECT images such that their volumes equal to the volumes of corresponding VOIs from contrast HCT-EX for Phantom \#2.

The tumor and NL segmentation protocol with least mean absolute error of TNR estimation would then be applied to SPECT reconstructed images using different AC protocols for TNR calculation.

\section{Dosimetric evaluation}

240 According to the Medical Internal Radiation Dose committee, the radiation dose from ${ }^{90} \mathrm{Y}$ resin 241 and glass microspheres to lungs, $\mathrm{D}_{\text {lung }}$, can be computed by the following equation:

$$
\mathrm{D}_{\text {lung }}[\mathrm{Gy}]=\frac{46.97 \times A_{0}[G B q] \times L S F}{M[k g]}
$$

where $A_{0}$ is the IA to the patient, i.e., assuming to be $3 \mathrm{GBq}$ for dosimetric calculation in this

244 study and $M$ is the lung mass. For planar images, lung mass was assumed to be $1 \mathrm{~kg}$ as suggested 245 [5] while for SPECT/CT images, it could be calculated by the product of the lung volume from 246 corresponding CT images and lung density, i.e., $0.3 \mathrm{~g} / \mathrm{cm}^{3}$ [28].

247 The radiation dose to the NL $\left(D_{N L}\right)$ and tumor $\left(D_{\text {tumor }}\right)$ could be estimated by Eq. 6 and Eq. 7 , 248 respectively. The mass of NL and tumor could be calculated by the product of their volumes 249 measured from the contrast HCT-EX image and soft tissue densities, i.e., $1.03 \mathrm{~g} / \mathrm{cm}^{3}$, 
respectively $[4,29]$. Only SPECT/CT date are used to calculate $\mathrm{D}_{N L}$ and $\mathrm{D}_{\text {tumor }}$ as TNR data are not available for the planar protocol.

$$
\begin{gathered}
\mathrm{D}_{N L}[\mathrm{~Gy}]=\frac{46.97 \times A_{0}[G B q] \times(1-L S F)}{\left(M_{\text {tumor }}[\mathrm{kg}] \times T N R+M_{N L}[\mathrm{~kg}]\right)} \\
\mathrm{D}_{\text {tumor }}[\mathrm{Gy}]=\frac{46.97 \times A_{0}[G B q] \times(1-L S F) \times T N R}{\left(M_{\text {tumor }}[\mathrm{kg}] \times T N R+M_{N L}[\mathrm{~kg}]\right)}
\end{gathered}
$$

\section{Injected activity calculation}

The partition model with three compartments of lungs, NL and tumor was used to calculate IA in this study [4]. To prevent the toxicity from lungs and NL, the maximum radiation dose to lungs in this study was set to 25 Gy while the maximum NL absorbed dose was 80 Gy respectively. After substituting the maximum lung absorbed dose into Eq. 5 to obtain the activity confined by lungs and the limited NL absorbed dose into Eq. 6 for the activity confined by NL, the lower activity value was selected as the IA.

The lung counts calculation in LSF, lung absorbed dose and IA calculations using Eq. 5 require the lung volume measurement which could be done using non-contrast CT of the SPECT/CT scan. Notwithstanding, it is challenging to measure lung volume in CACT and IACT due to their inherent motion blur (Fig. 3a, e). On the other hand, HCT-MID with clear organ contour (Fig. 3c) could provide sharper boundaries for volume measurement with similar lung size as compared to the corresponding SPECT. Therefore, in 4D CACT/IACT, one can extract one HCT-MID/interpolated CT at mid-respiration (ICT-MID), for lung VOI segmentation purpose. Such hybrid CT protocols were defined as CACT+HCT-MID and IACT+ICT-MID, where CACT and IACT were used for AC while the extracted HCT-MID and IACT/ICT-MID were used for lung VOI segmentation in LSF (and liver VOI segmentation if the estimation results of CT-based could be better than SPECT-based liver segmentation), lung absorbed dose 
272 and IA analysis to achieve better estimation. Additionally, as implied by Eqs. 6 and 7, the

273 absorbed dose calculation of NL and tumor is related to LSF and hence hybrid CT protocols are

274 applied.

275 The absolute errors of using planar and SPECT/CT protocols for LSF, TNR, lung, NL, tumor

276 absorbed dose and IA estimation for each phantom were calculated as compared to the ground

277 truth. A paired two-tailed t-test with Bonferroni correction was performed by SPSS Version 24

278 (IBM Corp., Armonk, NY, USA) between each protocol pair for different indices estimation

279 errors for 10 phantoms. A p value of $<0.05$ was regarded as statistically significant.

\section{Results}

\section{SPECT reconstruction images using different CT protocols}

283 Figure 9 shows the reconstructed images of Phantom \#9 with 20\% LSF, using different 284 attenuation correction protocols. Figure 10 illustrates the difference images between these 285 reconstructed images and the true activity map. Therein, white and black regions represent the 286 overestimation and underestimation of counts, respectively.

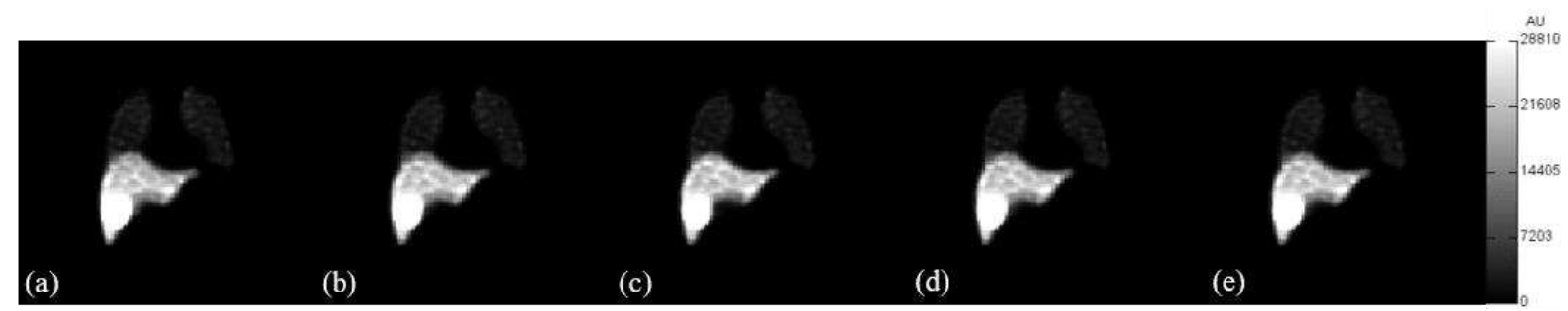

288 FIG. 9. Reconstructed images of Phantom \#9 with 20\% LSF using (a) HCT-IN, (b) HCT-MID,

(c) HCT-EX, (d) CACT and (e) IACT for AC. AU: Arbitrary unit. 


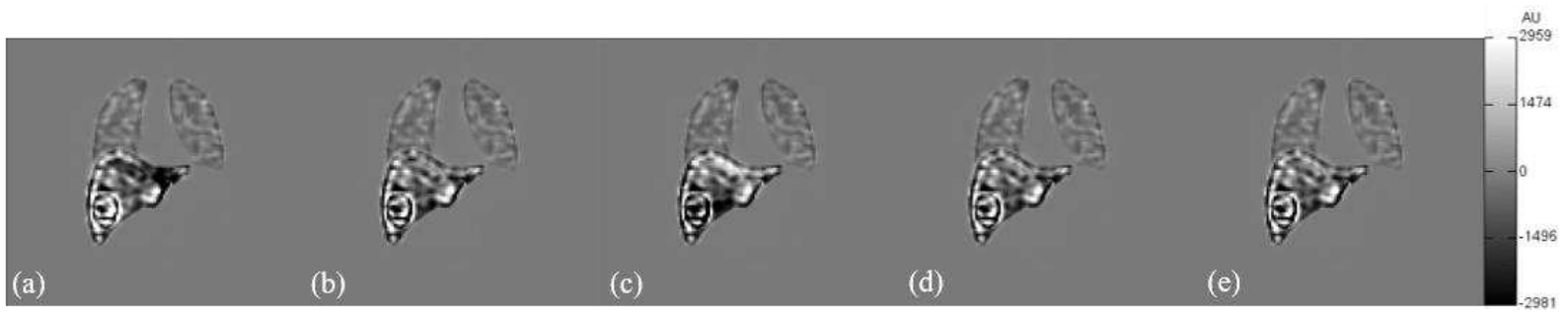

292 FIG. 10. The difference images between reconstructed images of Phantom \#9 with 20\% LSF 293 using (a) HCT-IN, (b) HCT-MID, (c) HCT-EX, (d) CACT and (e) IACT for AC and the true 294 activity map. AU: Arbitrary unit.

\section{LSF estimation}

297 The absolute errors (mean \pm deviation) of SPECT- and CT-based liver segmentation on LSF 298 estimation using different CT protocols for 10 phantoms are shown in Fig. 11. For SPECT/HCT299 IN, SPECT/CACT and SPECT/IACT, SPECT-based liver segmentation has lower errors than 300 CT-based liver segmentation. On contrary, CT-based liver segmentation has lower errors than 301 SPECT-based liver segmentation for SPECT/HCT-MID, SPECT/HCT-EX and hybrid CT 302 protocols, i.e., SPECT/CACT+HCT-MID and SPECT/IACT+ICT-MID where CACT/IACT is used for AC while HCT-MID/ICT-MID is used for segmentation. Generally, the mean absolute

304 errors for all protocols decrease along with the increase of LSF. The superior segmentation 305 method would be applied to the corresponding CT protocols to estimate LSF. 


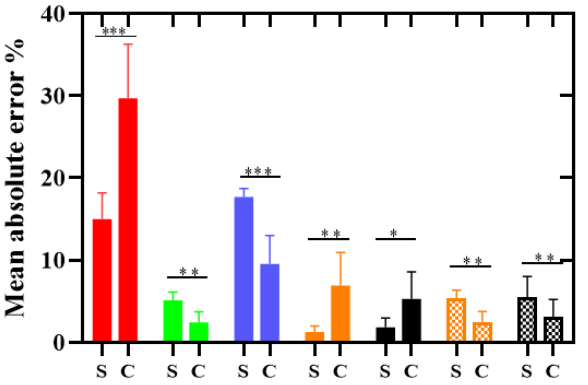

(a)

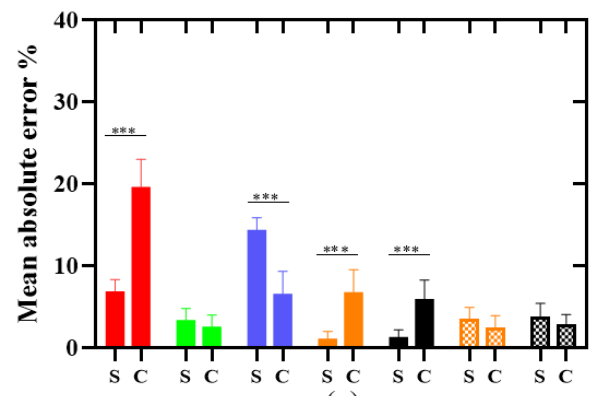

(c)

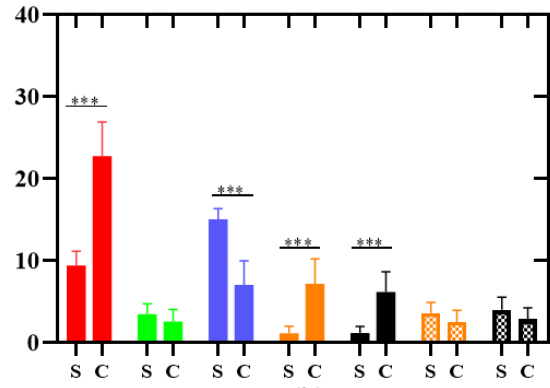

(b)

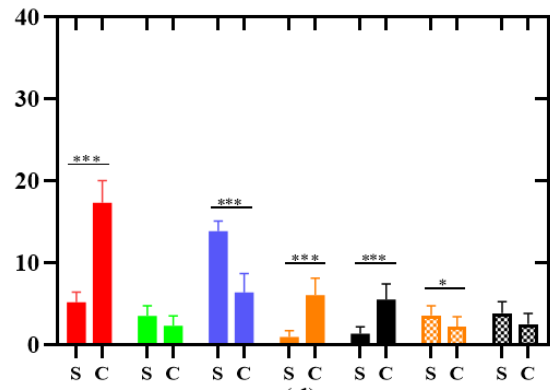

(d)
- SPECT/HCT-IN

- SPECT/HCT-MID

- SPECT/HCT-EX

- SPECT/CACT

- SPECT/IACT

SPECT/CACT+HCT-MID

m SPECT/IACT+ICT-MID

$*: \mathrm{p}<0.05$

$* *: \mathrm{p}<0.01$
307

FIG. 11. The mean absolute errors of LSF estimation for different CT protocols using SPECTand CT-based segmentation methods to segment liver for a LSF of (a) 5\%, (b) 10\%, (c) $15 \%$ and (d) 20\%, respectively. S: SPECT-based segmentation, C: CT-based segmentation.

The mean absolute errors of LSF estimation from planar and SPECT images with different CT protocols for AC and VOI segmentation are shown in Fig. 12. Planar images considerably overestimate the LSF at lower LSF, with $21.85 \pm 6.81 \%, 12.84 \pm 4.33 \%, 8.43 \pm 3.30 \%, 5.73 \pm 2.78 \%$ mean absolute errors for LSF of 5\%,10\%, 15\% and 20\%, respectively. SPECT/CACT has the best performance among all SPECT/CT protocols with the least mean absolute errors for LSF of $5 \% \quad(1.25 \pm 0.74 \%), \quad 10 \% \quad(1.13 \pm 0.88 \%), \quad 15 \% \quad(1.12 \pm 0.85 \%)$ and $20 \% \quad(0.95 \pm 0.762 \%)$. SPECT/IACT is slightly inferior to SPECT/CACT with mean absolute errors of $1.82 \pm 1.13 \%$, $1.20 \pm 0.77 \%, 1.30 \pm 0.87 \%$ and $1.35 \pm 0.83 \%$ for LSF of $5 \%, 10 \%, 15 \%$ and $20 \%$, respectively. SPECT/HCT-MID has the best performance out of SPECT/HCTs in LSF estimation. The errors from hybrid CT protocols, i.e., SPECT/CACT+HCT-MID and SPECT/IACT+ICT-MID, are 
about $1 \%$ higher than SPECT/CACT and SPECT/IACT respectively, and are similar to SPECT/HCT-MID. Generally, the mean absolute errors for all protocols decrease along with the increase of LSF. Except SPECT/HCT-EX, other SPECT/CTs and planar imaging overestimate the LSFs. The errors of planar and SPECT/HCT-IN protocols are statistically significantly higher than SPECT/CACT, SPECT/IACT, SPECT/HCT-MID and hybrid CT protocols for all LSFs.

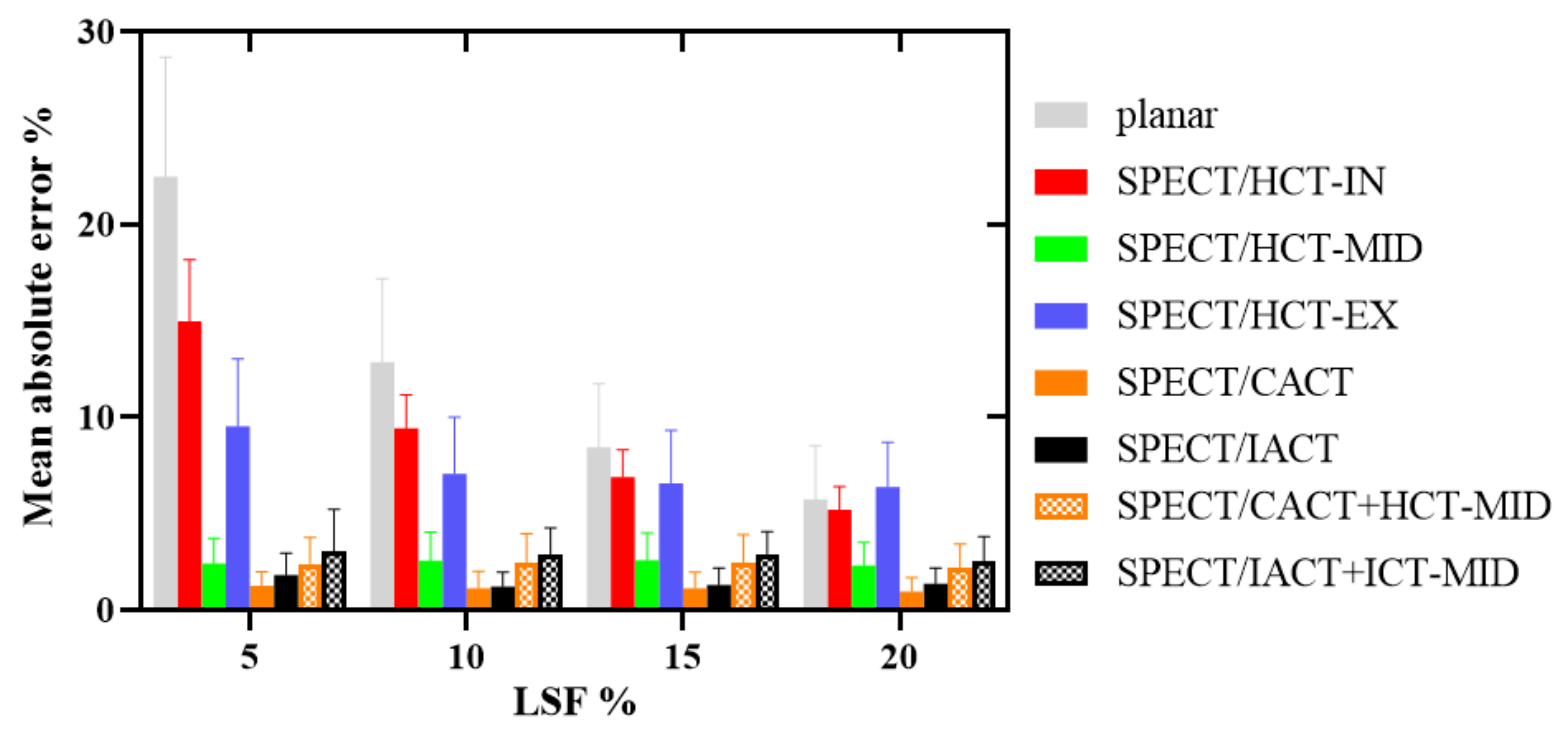

FIG. 12. The mean absolute errors of LSF estimation from SPECT images with different CT protocols for $\mathrm{AC}$ and VOI segmentation.

\section{TNR estimation}

For different tumor and NL segmentation protocols performed on SPECT/CACT images, their mean absolute errors are shown in Fig. 13. SPECT(50\%) segmentation protocol has the significantly lower mean absolute error of $8.43 \pm 5.00 \%$, which was later applied to SPECT reconstructed images using different $\mathrm{CT}$ protocols for $\mathrm{AC}$ to estimate the TNR in this study (Fig. 14). Results are similar for different LSFs thus only results for LSF of $5 \%$ are shown. SPECT/HCT-IN has the highest TNR errors of $10.42 \pm 5.65 \%$ while for other SPECT/CT images, 
their mean absolute errors of TNR estimation are similar, about $8.00 \pm 5.00 \%$. There is no

339 statistically significant difference between each AC protocol pair.

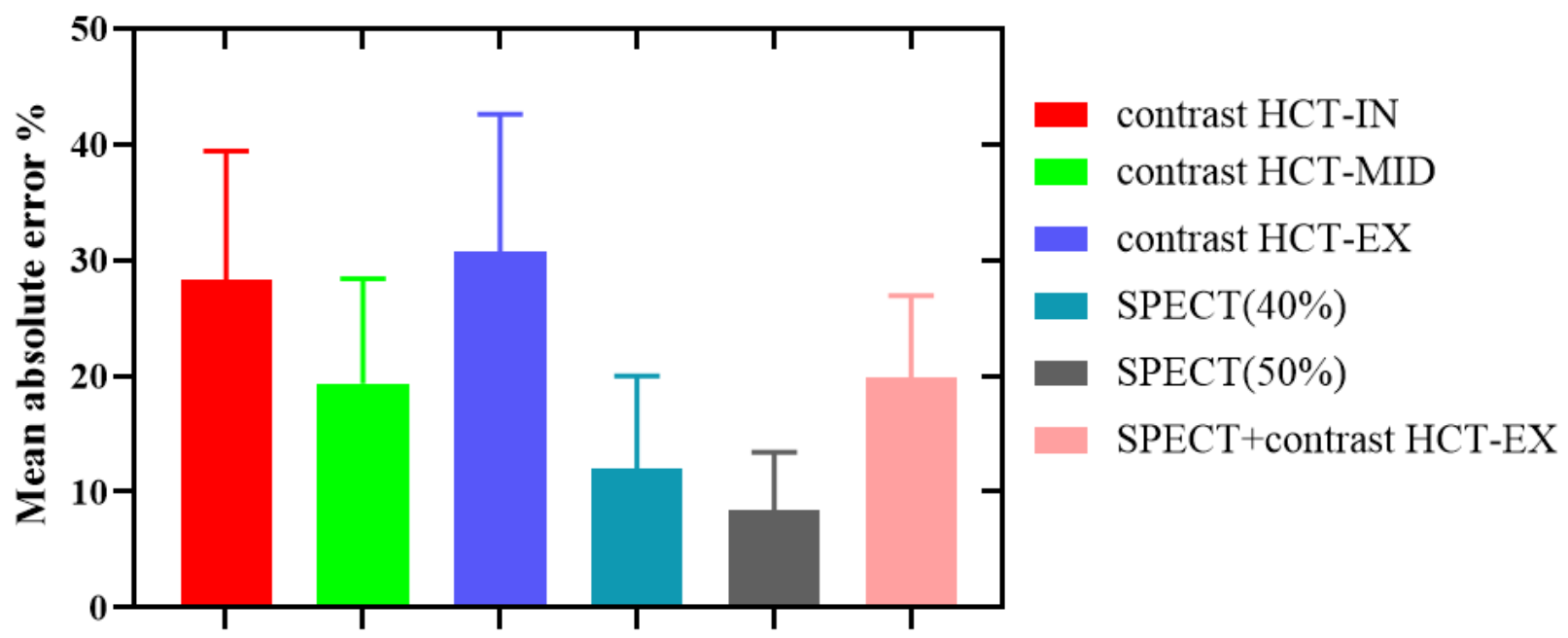

FIG. 13. The mean absolute errors of TNR estimation for different tumor and NL segmentation 342 methods for a LSF of $5 \%$.

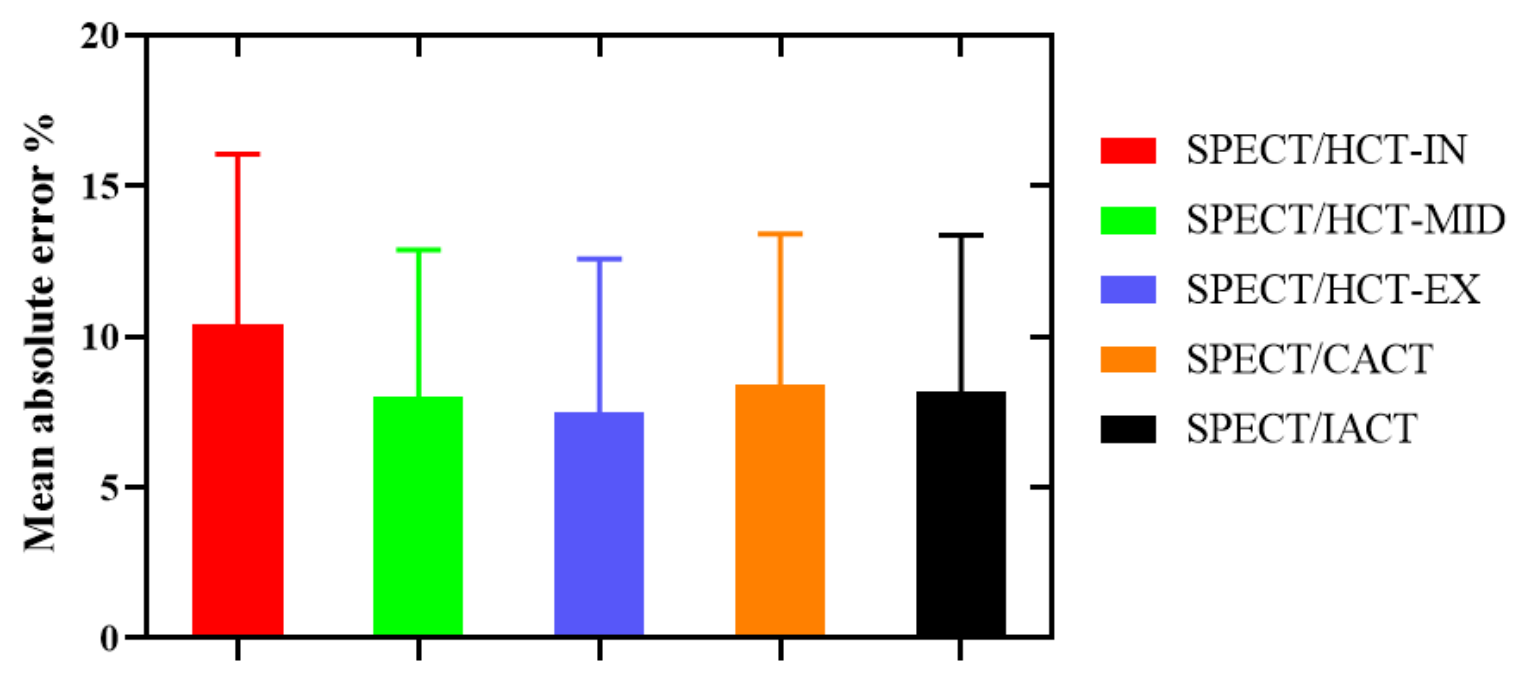

FIG. 14. The mean absolute errors of TNR estimation from SPECT images with different AC protocols using SPECT(50\%) segmentation method for a LSF of 5\%. 
The mean absolute errors for the lung absorbed dose are presented in Fig. 15. Compared to SPECT/CT protocols, planar images highly overestimate the lung absorbed dose, with mean absolute errors of up to $34.42 \pm 23.84 \%$ for LSF of $5 \%$, and their errors are statistically 351 significantly higher than those of SPECT/HCT-MID and hybrid CT protocols for all LSFs. On 352 the other hand, all SPECT/CT protocols underestimate lung absorbed dose except SPECT/HCT353 EX. SPECT/HCT-IN shows least mean absolute errors of lung absorbed dose for LSF of 5\% $354(2.97 \pm 1.80 \%)$ but its errors increase as the LSF increase. SPECT/HCT-MID and hybrid CT protocols have significantly lower errors for LSF of $10 \%(\sim 2.60 \pm 1.80 \%), 15 \%(\sim 2.50 \pm 1.80 \%)$, and $20 \%(\sim 2.50 \pm 1.50 \%)$ as compared to SPECT/HCT-IN, SPECT/CACT and SPECT/IACT.
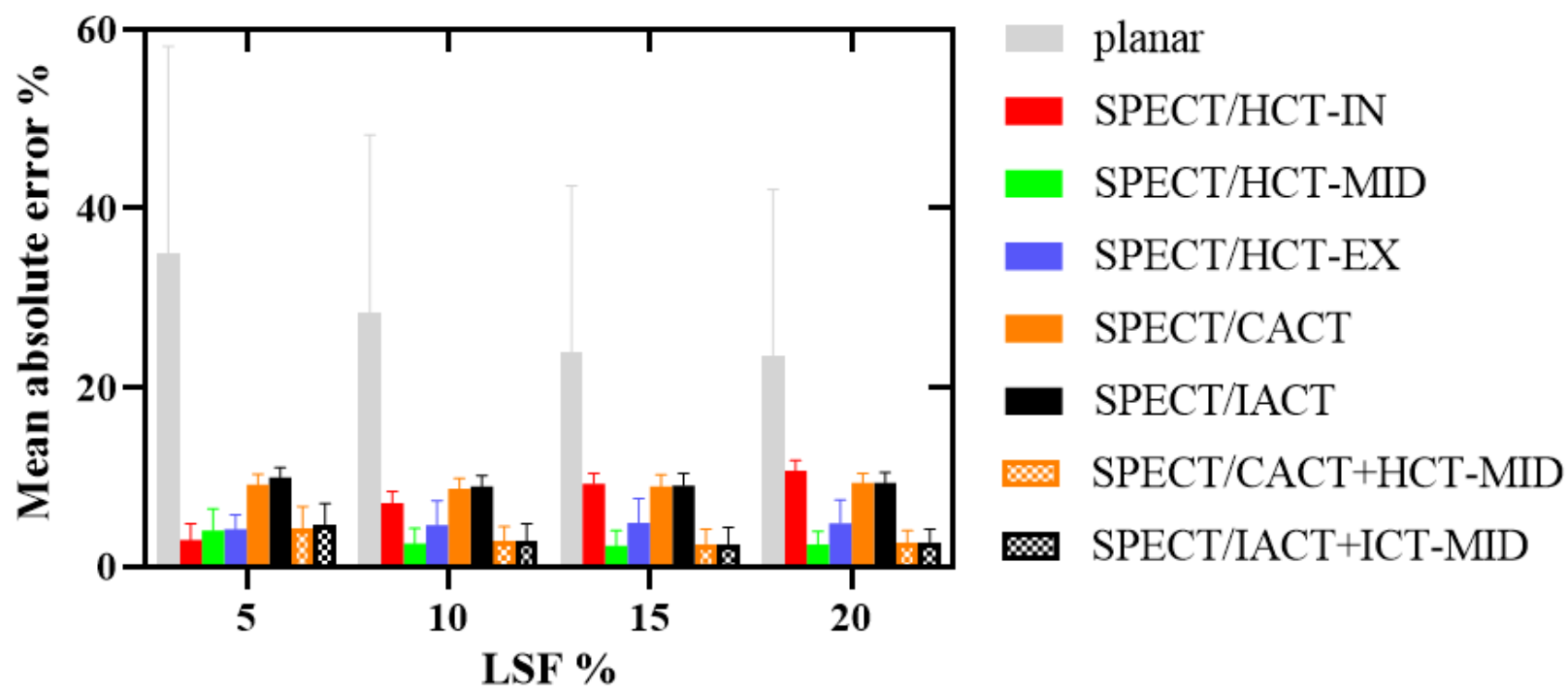

357 363 for all LSFs and protocols.

FIG. 15. The mean absolute errors of lung absorbed dose estimation for 10 phantoms.

For NL absorbed dose estimation, the mean and standard deviation of absolute errors from different CT protocols over all LSFs are similar $(\sim 2.00 \pm 2.00 \%)$ without significant difference and results of $\mathrm{LSF}=5 \%$ is shown in Fig. 16. It tends to be overestimated ( $~ 8$ out of 10 phantoms) 


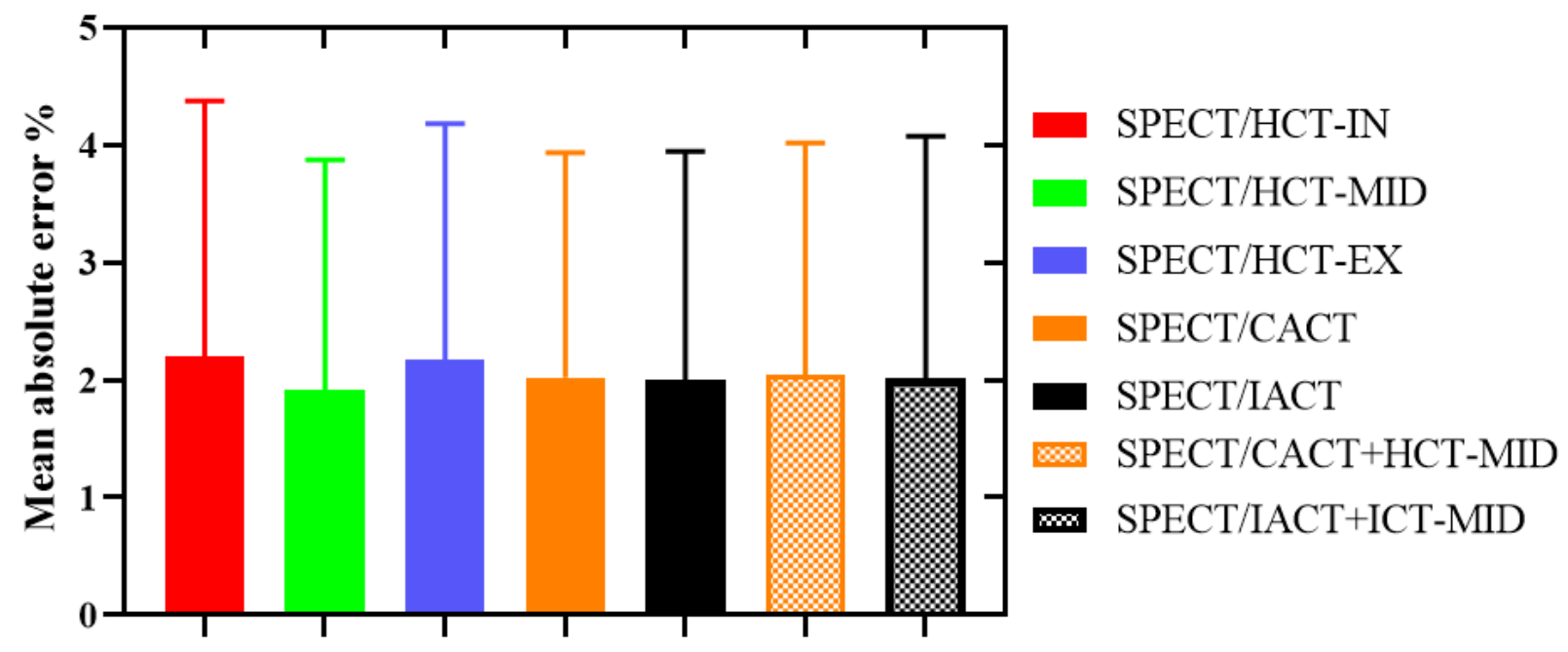

365 FIG. 16. The mean absolute errors of NL absorbed dose estimation for 10 phantoms with LSF of $3665 \%$.

368 For tumor absorbed dose estimation, the mean and standard deviation of absolute errors from 36910 phantoms with LSF of $5 \%$ is shown in Fig. 17. Results are also similar for different LSFs. 370 SPECT/HCT-IN has a slightly higher error with $8.41 \pm 5.24 \%$ than others $(\sim 6.00 \pm 4.00 \%)$. There 371 is no significant difference between each CT protocol pair. In contrast to NL absorbed dose, 372 tumor absorbed dose tends to be underestimated ( 8 out of 10 phantoms) for all LSFs and 373 protocols. 


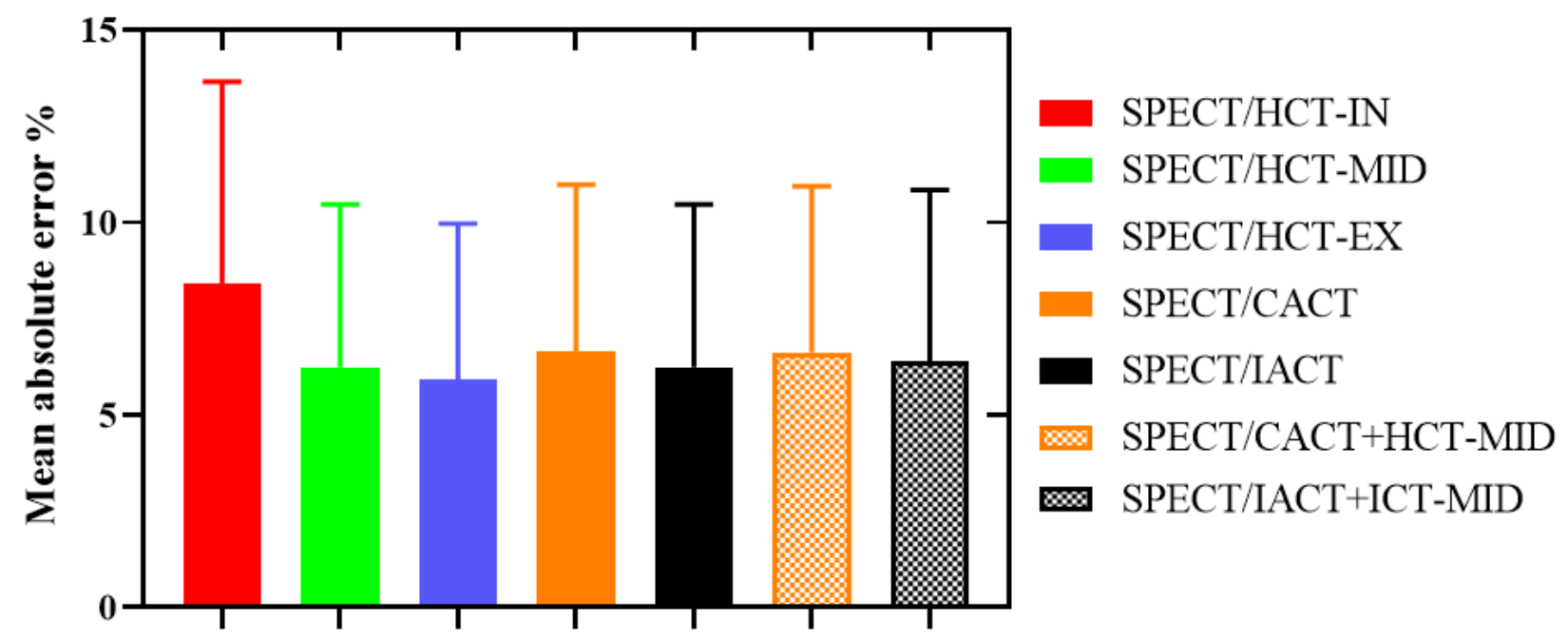

375 FIG. 17. The mean absolute errors of tumor absorbed dose estimation for 10 phantoms with LSF 376 of $5 \%$.

\section{Injected activity estimation}

379 Fig. 18 shows the mean and standard deviation of absolute errors of IA of 10 phantoms. The 380 errors of all SPECT/CT images with LSFs of 5\% and 10\% are generally lower than those with 381 LSFs of $15 \%$ and $20 \%$. For lower LSFs (LSF $\leq 10 \%)$, IA tends to be underestimated ( 8 out of 38210 phantoms), and all protocols have similar performance without statistically significant 383 difference. For LSF of $15 \%$ and $20 \%$, SPECT/HCT-IN results in highest IA estimation errors, 384 reaching $8.70 \pm 3.45 \%$ and $12.03 \pm 1.44 \%$ correspondingly while SPECT/HCT-MID and hybrid 385 CT protocols show statistically better results compared to other protocols. 


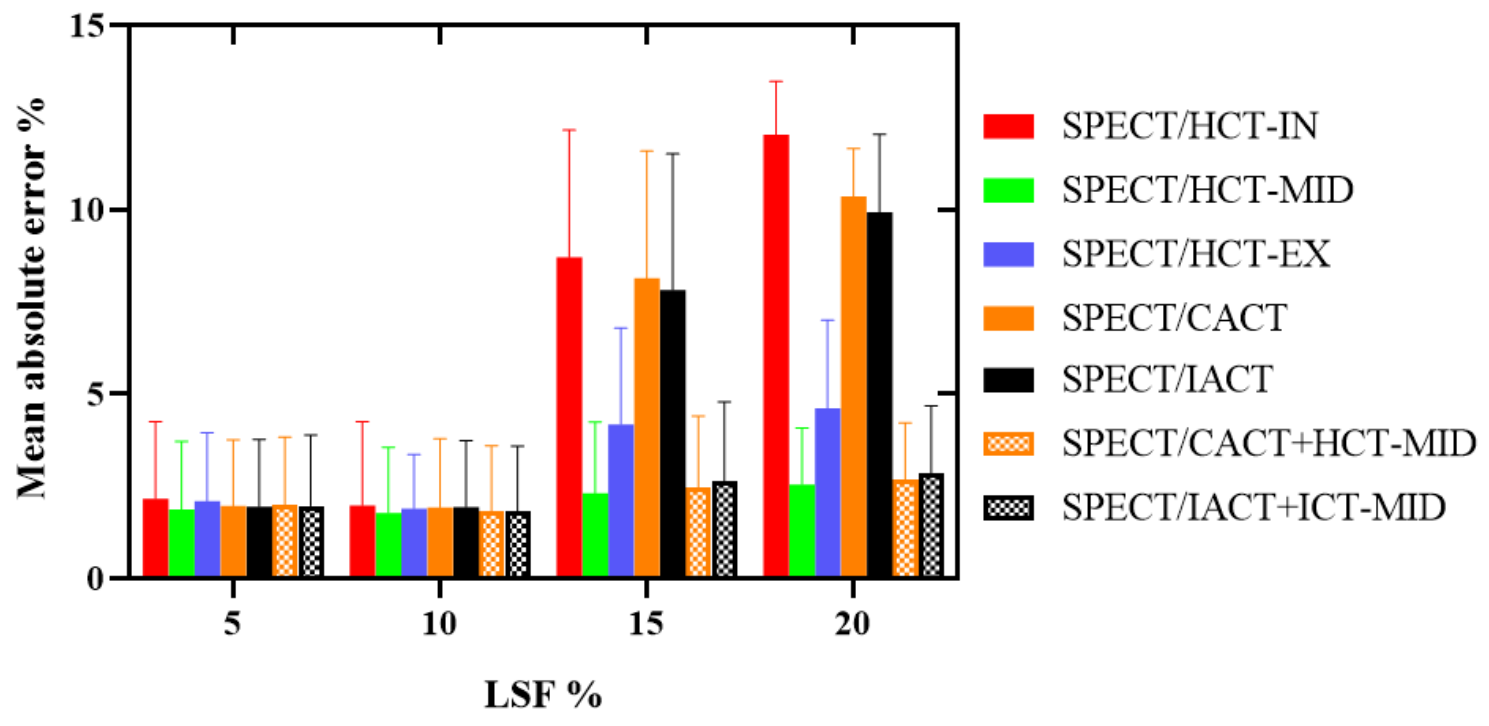

FIG. 18. The mean absolute errors of IA estimation from 10 phantoms.

Although Yu et al. [6] and Allred et al. [22] had already related AC to the accuracy of lung absorbed dose and LSF, they did not compare the effect of different AC maps in details.

Dittmann et al. [8] proposed using breath hold during CT and shallow breathing during SPECT to reduce the respiratory artifact in ${ }^{99 \mathrm{~m}} \mathrm{Tc}-\mathrm{MAA}$ SPECT, but there is a lack of clear criterion for

394 shallow breathing. Our study systemically evaluated various CT protocols for clinical ${ }^{99 \mathrm{~m}} \mathrm{Tc}-$ 395 MAA SPECT AC and segmentation reference. For HCT-IN, liver moves down due to lung 396 expansion resulting in underestimation of the top part of the liver activity (Fig. 10a) by 397 incorrectly using the attenuation coefficient of lungs for AC. For HCT-EX, the liver goes up 398 because of lung compression, leading to overestimation of the basal part of lungs activity (Fig. 399 10c) by incorrectly using the attenuation coefficient of the liver for AC. This problem can be 400 alleviated using HCT-MID as the AC map. Indeed, in the lung-liver interface on static SPECT, 
$\mathrm{AC}$ would require a mixture of lungs and liver attenuation coefficients in the lungs-liver border due to the motion blur. The average CTs, i.e., CACT and IACT, which average different states of respiration, can meet this requirement. Comparing with CACT, IACT is slightly inferior in image quality but can be generated with lower radiation exposure, with only two HCTs acquired in end-inspiration and end-expiration phases aided by an active breathing controller [14]. Our results, as expected, showed that compared to SPECT/CT images, ${ }^{99 \mathrm{~m}}$ Tc-MAA planar images overestimate the LSF even with $1 \mathrm{~cm}$ exclusion of ROIs in the lung-liver interface, probably attributed to the lack of AC, SC, CDR compensation and 3D information. The LSF errors from planar images without $1 \mathrm{~cm}$ exclusion of ROIs are about $10 \%$ higher than those with exclusion for each LSF setting (data not shown). The results are consistent with previous studies [6-8, 22]. Segmentation of lungs, liver and tumor is essential for both LSF, TNR and dosimetric analyses while breathing motion has significant impact on segmentation. For LSF estimation, the $2.21 \mathrm{~cm}$ exclusion segmentation method is suitable for HCT-MID, CACT and IACT to exclude the motion artifact. However, even with exclusion, HCT-IN tends to overestimate the lung volume while HCT-EX tends to underestimate it, which could be implied in Fig. 3(b) and (d). As a result, SPECT/HCT-IN overestimates the LSF while SPECT/HCT-EX underestimates it even with their own best liver segmentation method. Due to the motion blur, CACT and IACT also slightly overestimate the lung volume but they preserve the lung counts better than other methods. Meanwhile, the SPECT-based liver segmentation by setting lower threshold of $2.5 \%$ of maximum also preserves liver counts, resulting in good performance of CACT and IACT for LSF estimation. Thresholds of $2 \%, 2.5 \%, 3 \%, 3.5 \%$ and $4 \%$ of maximum intensity have been investigated and while threshold of $2.5 \%$ showed the best result thus is used in this study (data not shown). 
The use of SPECT with a good AC and segmentation protocol, i.e., CACT, IACT, HCT-MID or hybrid CT protocols, could possibly change the patient management as planar and SPECT/HCT-IN overestimates the LSFs, which may lead to prohibiting certain patients for the subsequent ${ }^{90} \mathrm{Y}$ radioembolization while SPECT/HCT-EX underestimates the LSFs, resulting in putting some patients at risk based on the 20\% LSF cutoff criteria [5]. Using mismatched AC maps and segmentation protocols can even lead to similar LSF errors as compared to planar for higher LSF, i.e., HCT-EX and HCT-IN for LSF $\geq 15 \%$.

For NL and tumor VOIs segmentation in TNR estimation, using SPECT images has proven to be more accurate than using contrast CT images in this study. However, the volume of NL and tumor VOIs segmented from SPECT images are inaccurate due to the inferior image quality. Thus, contrast CT images are utilized to perform the NL and tumor volume measurement in IA, NL and tumor absorbed dose calculation while the non-contrast CT for AC does not provide sufficient soft tissue contrast for these tasks. Contrast HCT-IN and HCT-EX are misaligned with SPECT images resulting in highest TNR estimation errors compared to other segmentation protocols. Meanwhile, contrast HCT-MID and SPECT+contrast HCT-EX segmentation protocols register better with SPECT images but the blurred boundary of the tumor on SPECT images due to respiration is included, leading to inaccurate tumor mean count density while SPECT image segmentation protocols with an appropriate threshold setting can alleviate this problem. In Fig. 14, different AC protocols for TNR estimation have similar performance given the best segmentation method, since the lung-liver interface accounting for a relatively small portion of the whole NL. Additionally, the SPECT-based segmentation method also reduces the impact of AC protocols for tumor mean count density estimation. The same trend was found for specific phantoms with tumor close to the lung-liver interface (data not shown). 
Dosimetric and IA estimation are affected by multiple factors, including LSF, compartment mass measurements and TNR according to the partition model, which make the results rather complex. For lung absorbed dose estimation, planar protocols are the worst compared to other SPECT/CT protocols due to inaccurate LSF estimation and standard lung mass assumption of 1 $\mathrm{kg}$. The error of average CTs is mainly due to incorrect lung mass measurement. Unexpectedly, generally, although they are both inaccurate in LSF and lung mass measurement. The concurrent overestimation of LSF and lung mass measurement from SPECT/HCT-IN and the underestimation of those from SPECT/HCT-EX result in lower lung absorbed dose error according to Eq. 5. As a result, the performance of SPECT/HCT-IN is not stable along with the changing of LSF. On the contrary, hybrid CT protocols and SPECT/HCT-MID are generally stable as a result of more accurate LSF and lung mass measurement. On the other hand, lung mass measurement may be further improved using lung CT densitovolumetry method proposed by Kao et al. [7].

For absorbed dose of NL and tumor, they are generally good due to low errors of TNR estimation as well as low errors of NL and tumor mass measurement from the contrast CT according to Eqs. 6 and 7. Moreover, the errors of NL absorbed dose are generally lower than those of tumor absorbed dose, attributed to a larger volume of NL that is less sensitive to volume measurement errors as compared to the tumor.

For IA estimation in Fig. 18, since the NL absorbed dose limitation is the dominant factor for low LSF $(<10 \%)$, the IA estimation error is generally low as a result of low errors of NL absorbed dose (Fig. 16). Along with the increase of LSF, the influence of LSF increases, leading to the similar order of IA errors for different CT protocols to that of lung absorbed dose errors, 
where the hybrid CT protocols and SPECT/HCT-MID provide the best results. Besides, lower LSF $(\leq 10 \%)$ is more common in the clinic $[8,22]$. Thus, different AC protocols are comparable for estimating IA.

The treatment planning results from HCT-MID and hybrid CT protocols are generally good and more stable compared to other protocols. However, similar to IACT, the HCT-MID acquisition would require the aid of an active breathing controller as it is difficult to have patients suspended their breath during mid-respiratory phase. In an ideal situation, shallow free breathing CT may have similar performance as compared to HCT-MID but that would highly depend on patients' compliance. Meanwhile, 4D CT protocols may be more feasible in clinic, where CACT could be used to perform AC in SPECT reconstruction while an HCT in middlerespiration phase could be extracted from CACT for VOI segmentation propose. However, higher radiation dose would be expected. If 4D CT or HCT-MID could not be realized, SPECT/HCT-EX is also a reasonable choice as compared to SPECT/HCT-IN and planar imaging. Respiratory motion affects the quantitative measurement in static ${ }^{99 \mathrm{~m}}$ Tc-MAA SPECT and could be potentially improved by respiratory gating method, which is currently under investigation in our laboratory. Moreover, recent studies pointed out that ${ }^{99 \mathrm{~m}}$ Tc-MAA was not perfectly reliable in modelling ${ }^{90} \mathrm{Y}$ microsphere distribution as the size and injected activity for ${ }^{99 \mathrm{~m}}$ Tc-MAA microspheres could be not exactly the same from ${ }^{90} \mathrm{Y}$ microspheres $\left({ }^{99 \mathrm{~m}} \mathrm{Tc}-\mathrm{MAA}\right.$ : mean diameter of 10-40 $\mu \mathrm{m}$, SIR-Sphere: mean diameter of 20-60 $\mu \mathrm{m}$, TheraSphere: mean diameter of $20-30 \mu \mathrm{m})[30,31]$. Therefore, ${ }^{90} \mathrm{Y}$ post-treatment imaging-based dosimetry should be conducted to confirm the parameters estimated by ${ }^{99 m}$ Tc-MAA. The effect of breathing motion, mismatch artifacts and segmentation accuracy may not have the same magnitude on ${ }^{90} \mathrm{Y}$ bremsstrahlung imaging/CT, as the image quality and quantitative accuracy are generally poor 
493 for bremsstrahlung imaging. However, ${ }^{90} \mathrm{Y}$ PET/CT provides higher image resolution [32, 33]

494 where respiratory motion, AC mismatch and segmentation could be more of a problem. Further 495 evaluation of our results using clinical ${ }^{99 \mathrm{~m}} \mathrm{Tc}-\mathrm{MAA}$ SPECT/CT, ${ }^{90} \mathrm{Y}$ bremsstrahlung imaging/CT

496 and ${ }^{90} \mathrm{Y}$ PET/CT patient data are beyond the scope of this study and need other independent 497 investigations.

498

499 Conclusions

500 The choice of CT in ${ }^{99 m}$ Tc-MAA SPECT/CT affects both AC and segmentation accuracy. Our 501 results showed that the LSF derived from ${ }^{99 \mathrm{~m}}$ Tc-MAA planar images is inferior to those obtained 502 from SPECT/CT images with good AC and segmentation methods. The LSF, TNR, absorbed 503 dose and IA estimation of ${ }^{99 \mathrm{~m}}$ Tc-MAA SPECT/CT could be further improved by the use of 4D 504 CT protocols in terms of $\mathrm{AC}$ and segmentation for personalized ${ }^{90} \mathrm{Y}$ microsphere treatment 505 planning. Helical CT acquired at end-expiration would also be a recommended choice if 4D CT 506 option is not available.

508 Abbreviations

509 AC: Attenuation correction; CACT: Cine average computed tomography; CDR: Collimator510 detector response; GM: Geometric mean; HCC: Hepatocellular carcinoma; HCT: Helical CT; 511 HCT-EX: Helical computed tomography acquired at end-expiration; HCT-IN: Helical computed 512 tomography acquired at end-inspiration; HCT-MID: Helical computed tomography acquired at 513 mid-respiration; HU: Hounsfield unit; IA: Injected activity; IACT: Interpolated average 514 computed tomography; ICT-MID: Interpolated computed tomography at mid-respiration; LEHR: 
515 Low energy high resolution; LSF: Lung shunting fraction; NL: Normal liver; NSD: Normalized

516 standard deviation; OS-EM: Ordered subset expectation maximization; PET/CT: Positron

517 emission tomography/computed tomography; ROI: Region-of-interest; SC: Scatter correction;

518 SIRT: Selective internal radiation therapy; SPECT/CT: Single-photon emission computed

519 tomography/computed tomography; TNR: Tumor-to-normal liver ratio; ${ }^{99 \mathrm{~m}} \mathrm{Tc}-\mathrm{MAA}:{ }^{99 \mathrm{~m}} \mathrm{Tc}-$

520 macroaggregated albumin; VOI: Volume-of-interest; XCAT: 4D digital extended cardiac torso

522 Acknowledgements

523 We thank former lab members, Drs. Duo Zhang and Ilker Ozsahin for proofreading the 524 manuscript, and Mr. Jianying Lee from Taipei Veterans General Hospital for providing clinical 525 information.

\section{Authors' contributions}

528

529

530

531

$532 \quad$ Funding

533 This work was supported by the research grant from University of Macau (MYRG2017-00060534 FST).

535

\section{$536 \quad$ Availability of data and materials}

537 The datasets used and analyzed during the current study are available from the corresponding 
author on reasonable request.

540 Ethics approval and consent to participate

$541 \quad$ Not applicable.

542

543 Consent for publication

544 Not applicable.

545

546 Competing interests

547 The authors declare that they have no competing interests.

\section{Reference}

550 1. Salem R, Lewandowski RJ, Kulik L, Wang E, Riaz A, Ryu RK, et al. Radioembolization results in longer

551 time-to-progression and reduced toxicity compared with chemoembolization in patients with hepatocellular 552 carcinoma. Gastroenterology. 2011;140:497-507 e2.

553 2. Sarfaraz M, Kennedy AS, Cao ZJ, Sackett GD, Yu CX, Lodge MA, et al. Physical aspects of yttrium-90 554 microsphere therapy for nonresectable hepatic tumors. Med Phys. 2003;30:199-203.

555 3. Riaz A, Awais R, Salem R. Side effects of yttrium-90 radioembolization. Front Oncol. 2014;4:198. 556 https://doi.org/10.3389/fonc.2014.00198.

557 4. Ho S, Lau WY, Leung TW, Chan M, Ngar YK, Johnson PJ, et al. Partition model for estimating radiation 558 doses from yttrium-90 microspheres in treating hepatic tumours. Eur J Nucl Med. 1996;23:947-52.

$559 \quad$ 5. Garin E, Rolland Y, Laffont S, Edeline J. Clinical impact of (99m)Tc-MAA SPECT/CT-based dosimetry in 560 the radioembolization of liver malignancies with (90)Y-loaded microspheres. Eur J Nucl Med Mol Imaging. 561 2016;43:559-75. https://doi.org/10.1007/s00259-015-3157-8. 
562 6. Yu N, Srinivas SM, Difilippo FP, Shrikanthan S, Levitin A, McLennan G, et al. Lung dose calculation with 563 SPECT/CT for (90)Yittrium radioembolization of liver cancer. Int J Radiat Oncol Biol Phys. 2013;85:834-9.

564 7. Kao YH, Magsombol BM, Toh Y, Tay KH, Chow P, Goh AS, et al. Personalized predictive lung dosimetry 565 by technetium-99m macroaggregated albumin SPECT/CT for yttrium-90 radioembolization. EJNMMI Res. 566 2014;4:33. https://doi.org/10.1186/s13550-014-0033-7.

567 8. Dittmann H, Kopp D, Kupferschlaeger J, Feil D, Groezinger G, Syha R, et al. A Prospective Study of 568 Quantitative SPECT/CT for Evaluation of Lung Shunt Fraction Before SIRT of Liver Tumors. J Nucl Med. $569 \quad 2018 ; 59: 1366-72$.

5709 9. Segars WP, Tsui BM, editors. Effect of respiratory motion in CT-based attenuation correction in SPECT 571 using different CT scanners and protocols. IEEE Nuclear Science Symposium Conference Record. 2005;doi: 572 10.1109/NSSMIC.2005.1596819.

573 10. McQuaid SJ, Hutton BF. Sources of attenuation-correction artefascts in cardiac PET/CT and SPECT/CT. 574 Eur J Nucl Med Mol Imaging. 2008;35:1117-23.

575 11. Zhang D, Yang BH, Wu NY, Mok GS. Respiratory average CT for attenuation correction in myocardial 576 perfusion SPECT/CT. Ann Nucl Med. 2017;31:172-80.

577 12. Zhang D, Ghaly M, Mok GSP. Interpolated CT for attenuation correction on respiratory gating cardiac 578 SPECT/CT - A simulation study. Med Phys. 2019;46:2621-8.

579 13. Mok GS, Ho CY, Yang B-H, Wu T-H. Interpolated average CT for cardiac PET/CT attenuation correction. $580 \quad$ J Nucl Cardiol. 2016;23:1072-9.

581 14. Sun T, Wu TH, Wang SJ, Yang BH, Wu NY, Mok GS. Low dose interpolated average CT for thoracic 582 PET/CT attenuation correction using an active breathing controller. Med Phys. 2013;40:102507.

583 15. Ho CY, Wu T-H, Mok GS. Interpolated average CT for PET attenuation correction in different lesion 584 characteristics. Nucl Med Commun. 2016;37:297-306.

585 16. Bastiaannet R, Kappadath SC, Kunnen B, Braat AJAT, Lam MGEH, de Jong HWAM. The physics of 586 radioembolization. EJNMMI Physics. 2018;5:22. https://doi.org/10.1186/s40658-018-0221-z.

587 17. Segars WP, Sturgeon G, Mendonca S, Grimes J, Tsui BM. 4D XCAT phantom for multimodality imaging 588 research. Med Phys. 2010;37:4902-15. 

myocardial perfusion SPECT imaging research. Phys Med Biol. 2014;59:2935.

591 19. Ho CL, Chen S, Cheung SK, Leung YL, Cheng KC, Wong KN, et al. Radioembolization with (90)Y glass 592 microspheres for hepatocellular carcinoma: significance of pretreatment (11)C-acetate and (18)F-FDG PET/CT and posttreatment (90)Y PET/CT in individualized dose prescription. Eur J Nucl Med Mol Imaging. 2018;45:2110-21.

594 20. Frey E, Tsui B. A practical method for incorporating scatter in a projector-backprojector for accurate 595 scatter compensation in SPECT. IEEE Transactions on Nuclear Science. 1993;40:1107-16.

596 21. Frey EC, Tsui B, editors. A new method for modeling the spatially-variant, object-dependent scatter 597 response function in SPECT. 1996 IEEE Nuclear Science Symposium Conference Record. 1996;doi: $598 \quad 10.1109 /$ NSSMIC.1996.591559.

599 22. Allred JD, Niedbala J, Mikell JK, Owen D, Frey KA, Dewaraja YK. The value of (99m)Tc-MAA 600 SPECT/CT for lung shunt estimation in (90)Y radioembolization: a phantom and patient study. EJNMMI Res. $601 \quad 2018 ; 8: 50$. https://doi.org/10.1186/s13550-018-0402-8.

602 23. Mok GS, Sun T, Huang TC, Vai MI. Interpolated average CT for attenuation correction in PET--a 603 simulation study. IEEE Trans Biomed Eng. 2013;60:1927-34.

604 24. Huang TC, Mok GS, Wang SJ, Wu TH, Zhang G. Attenuation correction of PET images with interpolated 605 average CT for thoracic tumors. Phys Med Biol. 2011;56:2559-67.

606 25. Yushkevich PA, Piven J, Hazlett HC, Smith RG, Ho S, Gee JC, et al. User-guided 3D active contour 607 segmentation of anatomical structures: significantly improved efficiency and reliability. Neuroimage. 2006;31:111660828.

609 26. Bastiaannet R, Viergever MA, de Jong HW. Impact of respiratory motion and acquisition settings on 610 SPECT liver dosimetry for radioembolization. Med Phys. 2017;44:5270-9.

611 27. Chansanti O, Jahangiri Y, Matsui Y, Adachi A, Geeratikun Y, Kaufman JA, et al. Tumor Dose Response in 612 Yttrium-90 Resin Microsphere Embolization for Neuroendocrine Liver Metastases: A Tumor-Specific Analysis with 613 Dose Estimation Using SPECT-CT. J Vasc Interv Radiol. 2017;28:1528-35.

614 28. Van Dyk J, Keane TJ, Rider WD. Lung density as measured by computerized tomography: implications for 615 radiotherapy. Int J Radiat Oncol Biol Phys. 1982;8:1363-72. 
616 29. Gulec SA, Mesoloras G, Stabin M. Dosimetric techniques in 90Y-microsphere therapy of liver cancer: The 617 MIRD equations for dose calculations. J Nucl Med. 2006;47:1209-11.

618 30. Elschot M, Nijsen JF, Lam MG, Smits ML, Prince JF, Viergever MA, et al. (99m)Tc-MAA overestimates 619 the absorbed dose to the lungs in radioembolization: a quantitative evaluation in patients treated with (166)Ho620 microspheres. Eur J Nucl Med Mol Imaging. 2014;41:1965-75.

621 31. Wondergem M, Smits ML, Elschot M, de Jong HW, Verkooijen HM, van den Bosch MA, et al. 99mTc622 macroaggregated albumin poorly predicts the intrahepatic distribution of $90 \mathrm{Y}$ resin microspheres in hepatic 623 radioembolization. J Nucl Med. 2013;54:1294-301. https://doi.org/10.2967/jnumed.112.117614.

624 32. Yue J, Mauxion T, Reyes DK, Lodge MA, Hobbs RF, Rong X, et al. Comparison of quantitative Y-90 625 SPECT and non-time-of-flight PET imaging in post-therapy radioembolization of liver cancer. Med Phys. $6262016 ; 43: 5779$.

627 33. Elschot M, Vermolen BJ, Lam MG, de Keizer B, van den Bosch MA, de Jong HW. Quantitative 628 comparison of PET and Bremsstrahlung SPECT for imaging the in vivo yttrium-90 microsphere distribution after 629 liver radioembolization. PLoS One. 2013;8:e55742. https://doi.org/10.1371/journal.pone.0055742. 


\section{Figures}

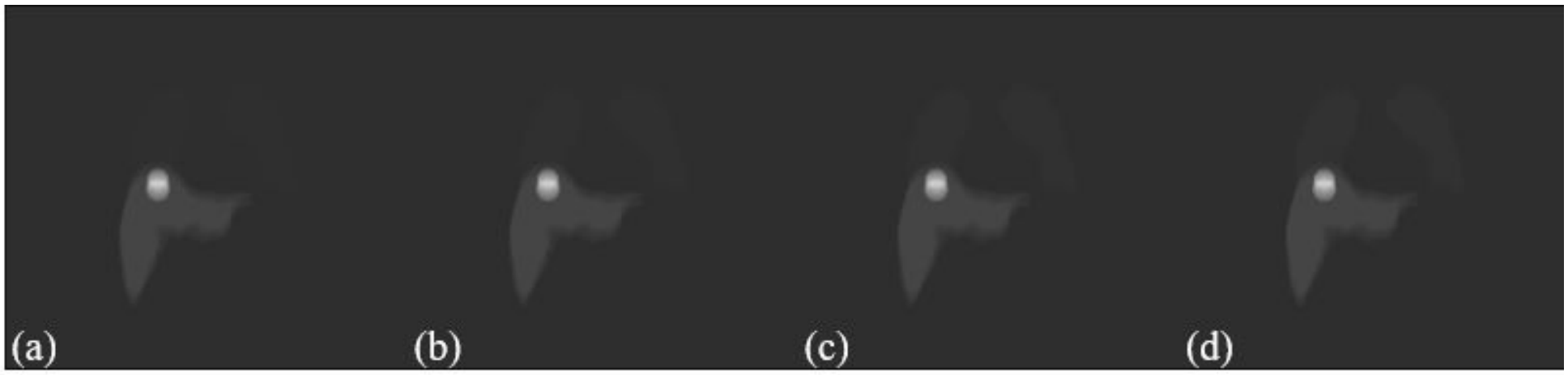

\section{Figure 1}

Phantom \#6 with LSF of (a) $5 \%$, (b) $10 \%$, (c) $15 \%$ and (d) $20 \%$.

\section{Figure 2}

Noisy anterior (left) and posterior (right) planar images of Phantom \#3.

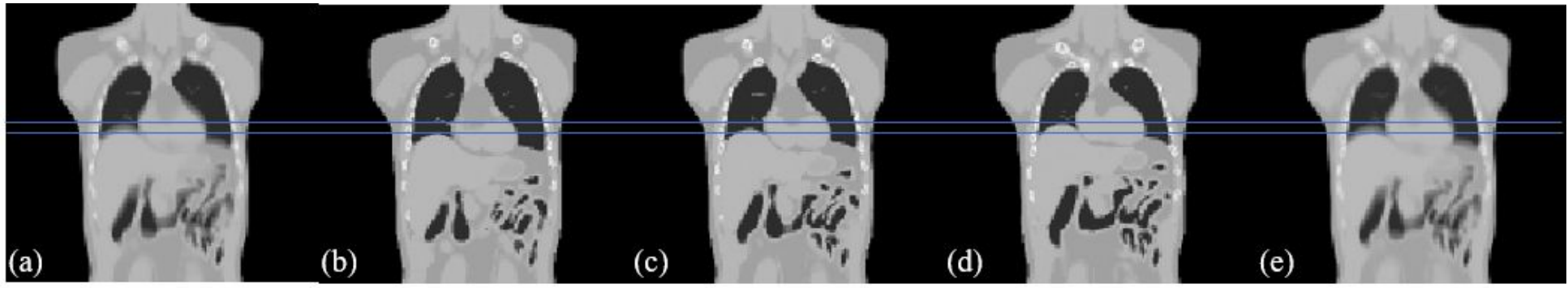

Figure 3

(a) CACT, (b) HCT-IN, (c) HCT-MID, (d) HCT-EX and (e) IACT of Phantom \#1. Blue lines represent motion range of $2 \mathrm{~cm}$. 

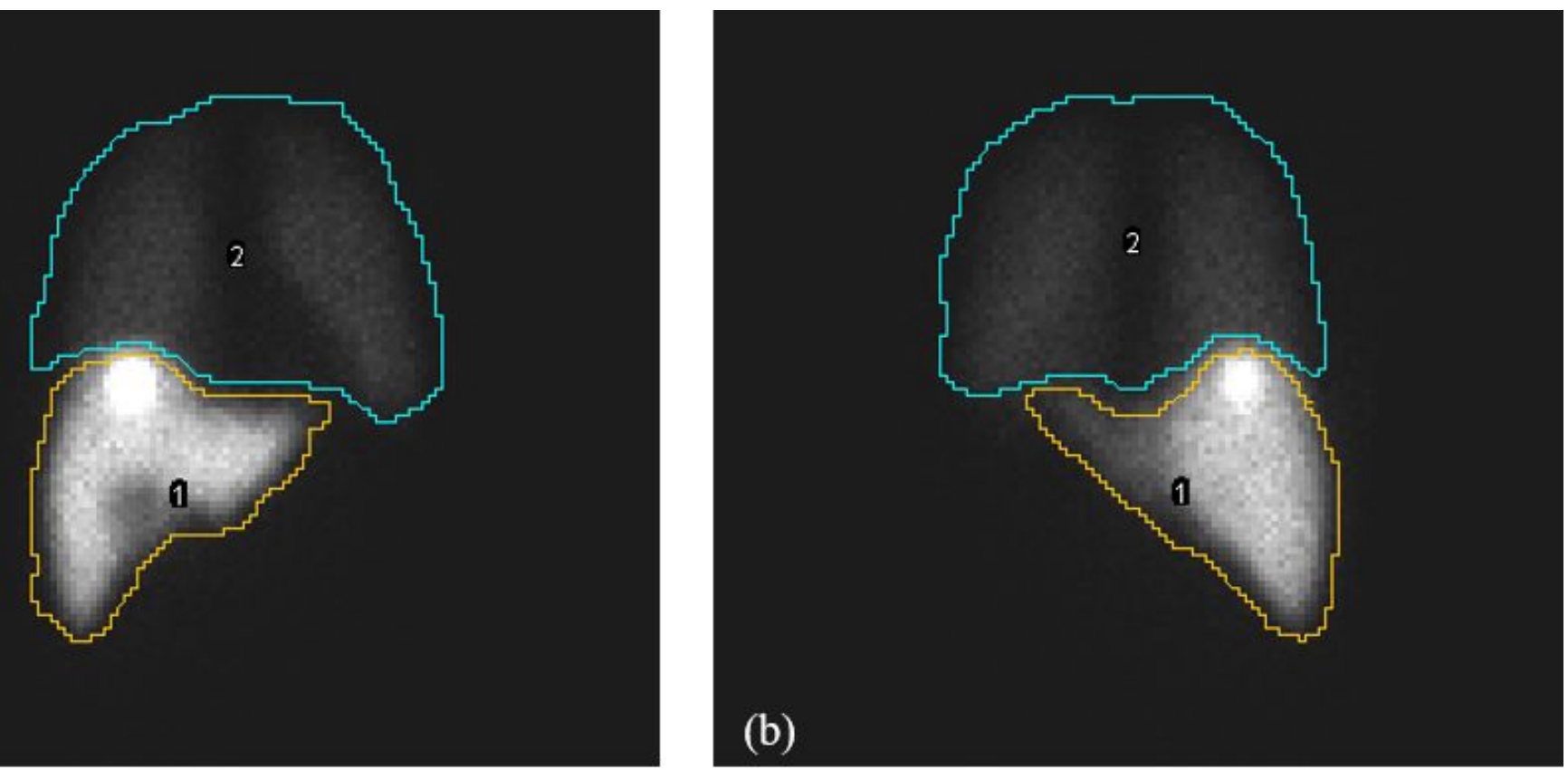

(a)

\section{Figure 4}

Liver (orange ROI) and lung segmentation (blue ROI) of planar images on (a) anterior and (b) posterior view.
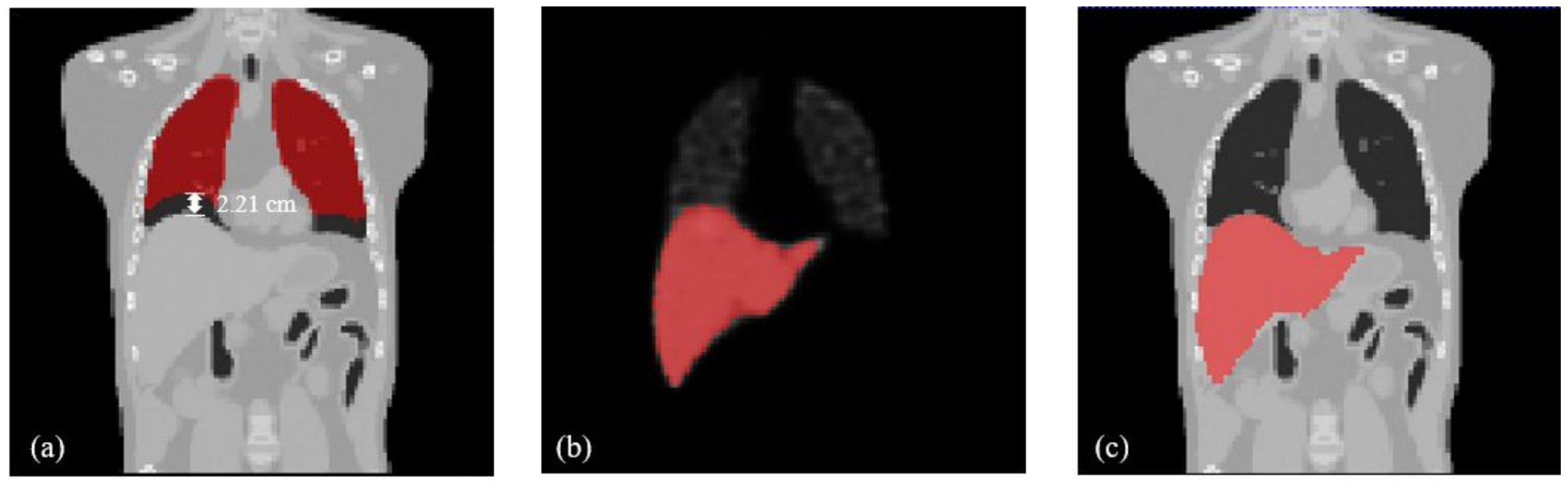

Figure 5

(a) Lung segmentation with $2.21 \mathrm{~cm}$ axial exclusion on HCT-MID.(b) Liver segmentation on SPECT image and on (c) HCT-MID for Phantom \#1. 

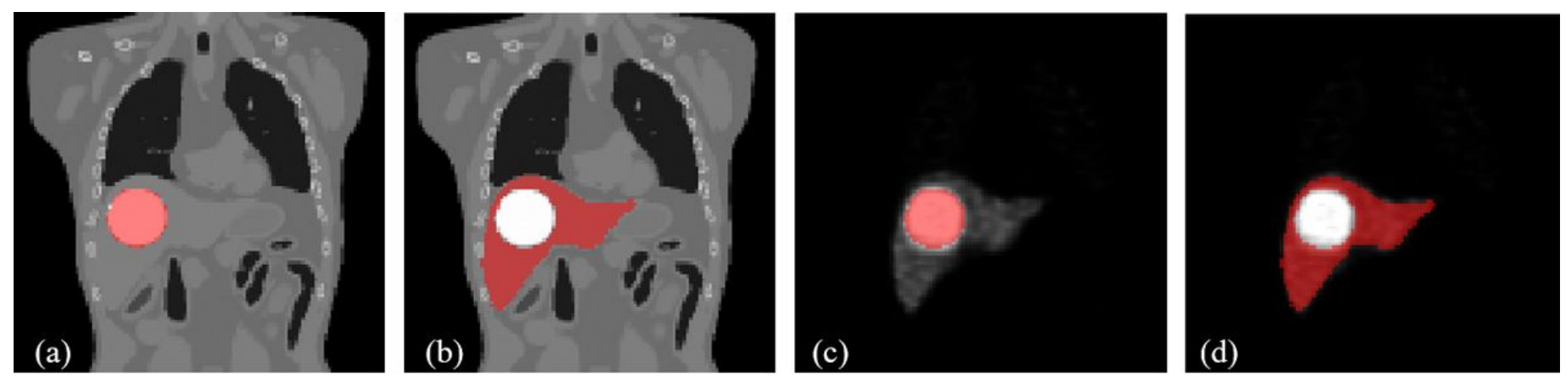

\section{Figure 6}

(a) Tumor and (b) NL segmentation on contrast HCT-MID. Corresponding VOIs were then mapped to segment the (c) tumor and (d) NL on SPECT images for Phantom \#2.
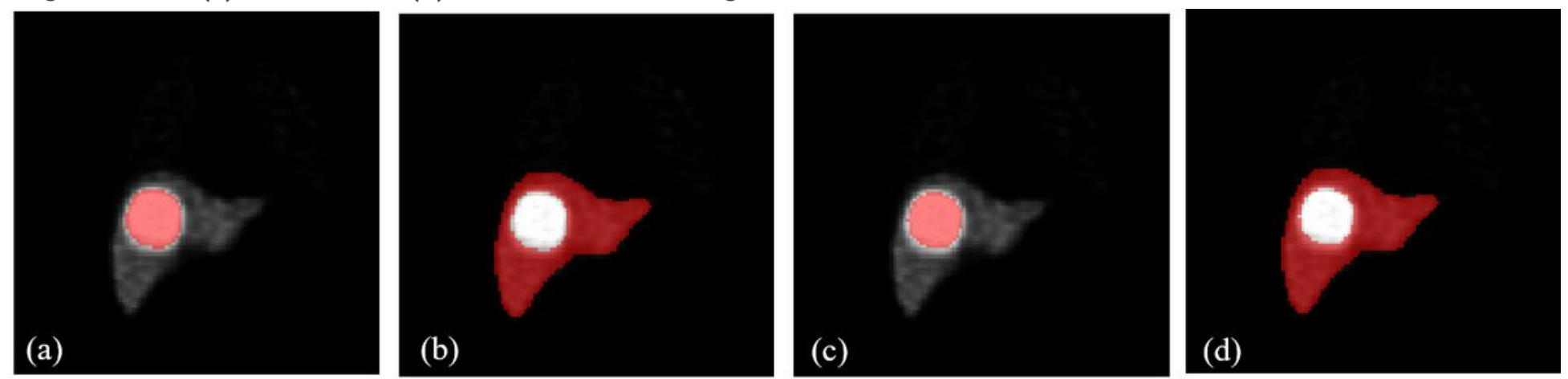

\section{Figure 7}

(a) Tumor segmented from $40 \%$ of maximum intensity and (b) corresponding NL VOI. (c) Tumor segmented from $50 \%$ of maximum intensity and (d) corresponding NL VOI on SPECT images for Phantom \#2.
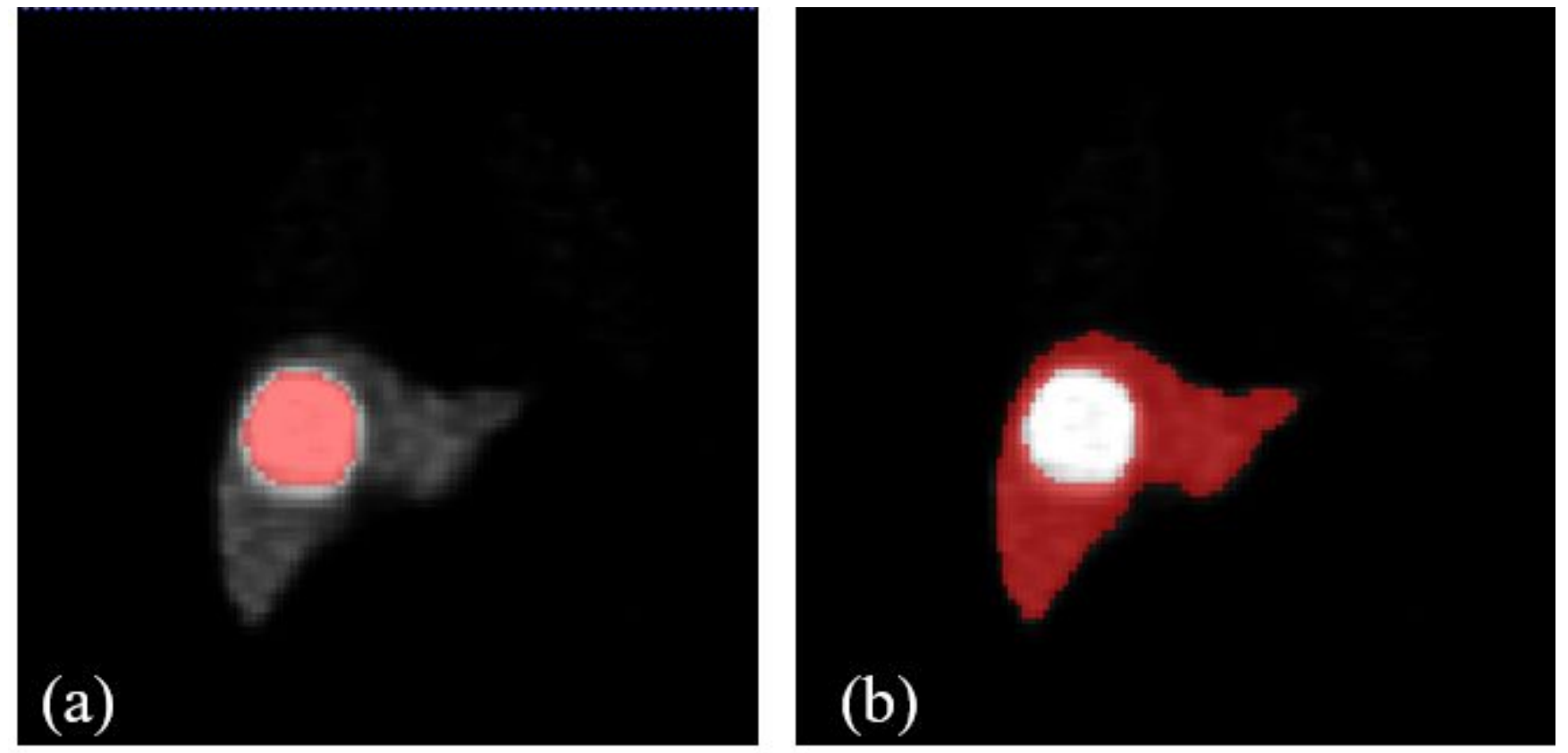


\section{Figure 8}

(a) Tumor and (b) NL VOIs on SPECT images such that their volumes equal to the volumes of corresponding VOls from contrast HCT-EX for Phantom \#2.

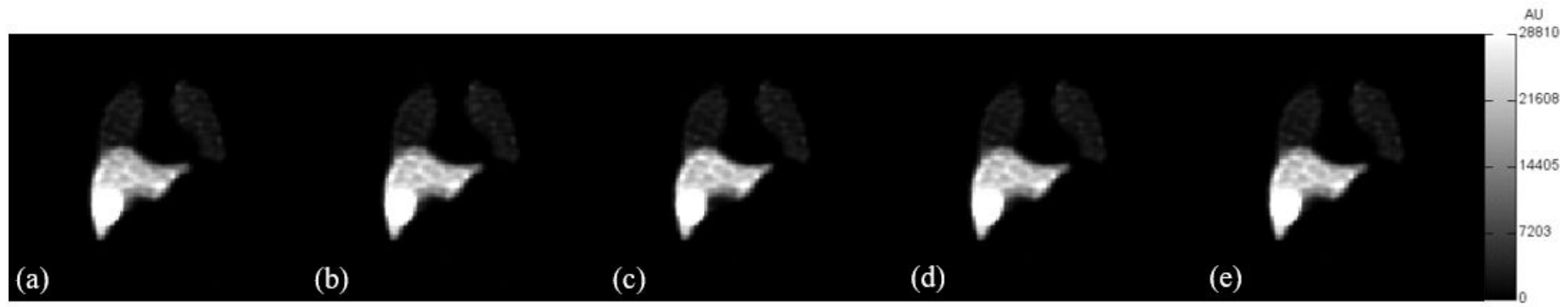

\section{Figure 9}

Reconstructed images of Phantom \#9 with 20\% LSF using (a) HCT-IN, (b) HCT-MID, (c) HCT-EX, (d) CACT and (e) IACT for AC. AU: Arbitrary unit.

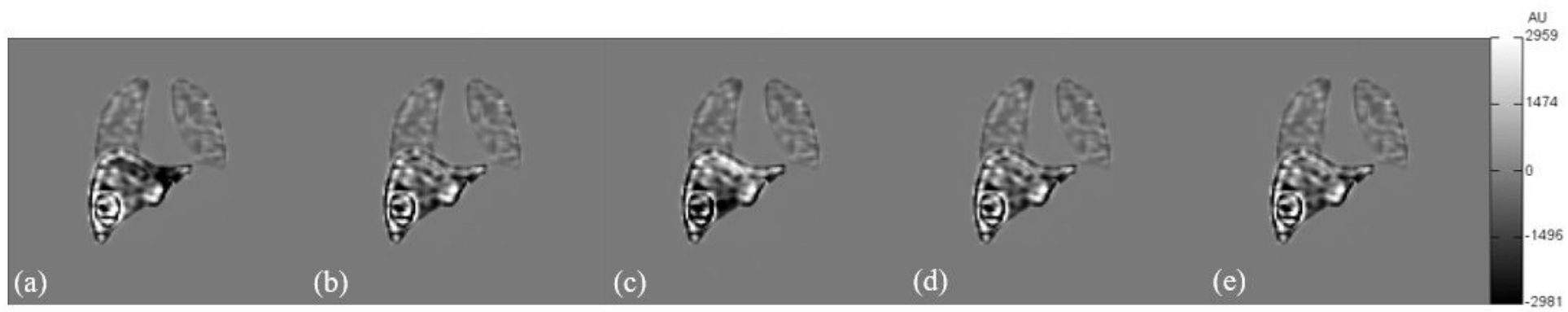

\section{Figure 10}

The difference images between reconstructed images of Phantom \#9 with $20 \%$ LSF using (a) HCT-IN, (b) HCT-MID, (c) HCT-EX, (d) CACT and (e) IACT for AC and the true activity map. AU: Arbitrary unit. 


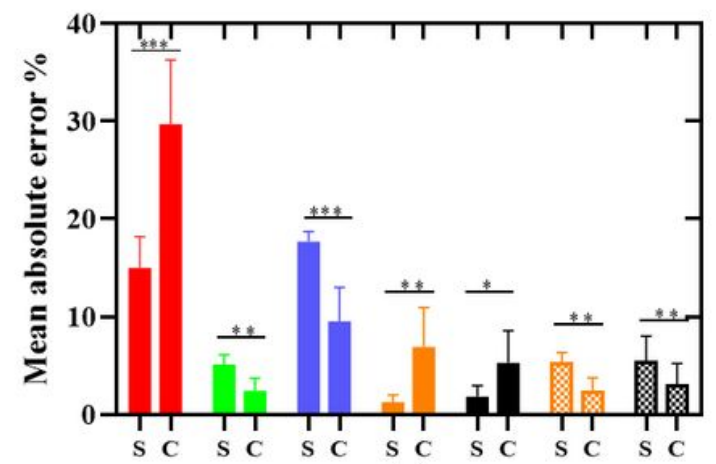

(a)

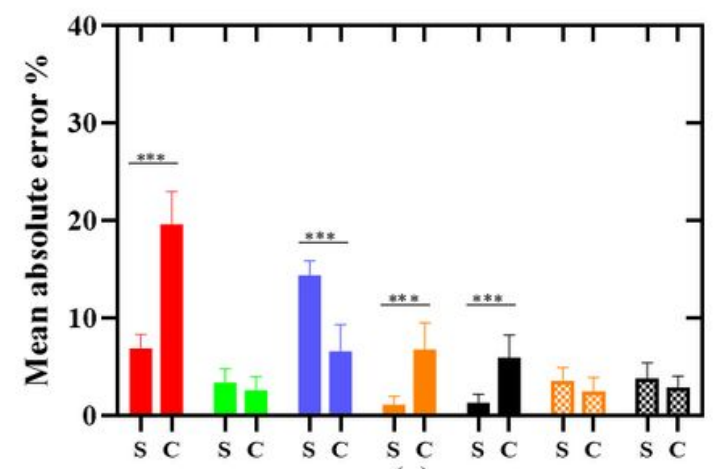

(c)

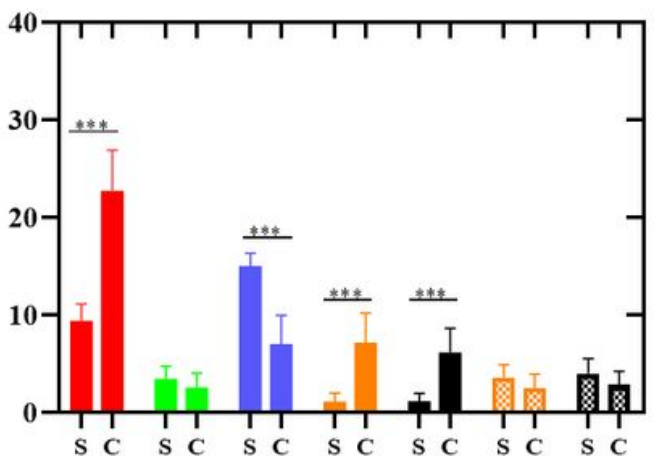

(b)

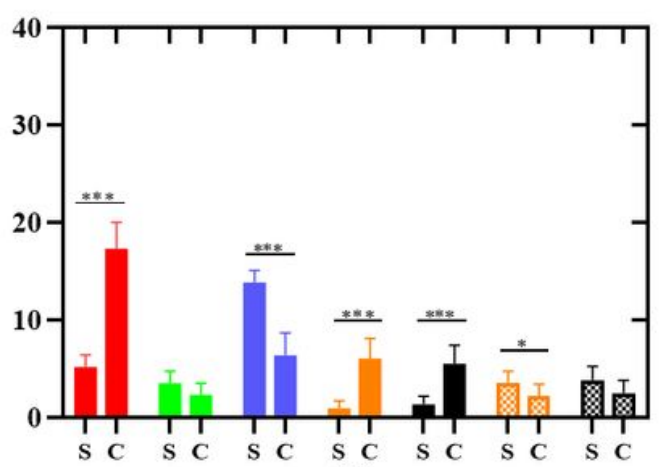

(d)
SPECT/HCT-IN

- SPECT/HCT-MID

- SPECT/HCT-EX

SPECT/CACT

- SPECT/IACT

SPECT/CACT + HCT-MID

sPECT/IACT+ICT-MID

$*: \mathrm{p}<0.05$

$* *: \mathrm{p}<0.01$

$* * *: \mathrm{p}<0.001$

\section{Figure 11}

The mean absolute errors of LSF estimation for different CT protocols using SPECT- and CT-based segmentation methods to segment liver for a LSF of (a) $5 \%$, (b) $10 \%$, (c) $15 \%$ and (d) $20 \%$, respectively. S: SPECT-based segmentation, C: CT-based segmentation.

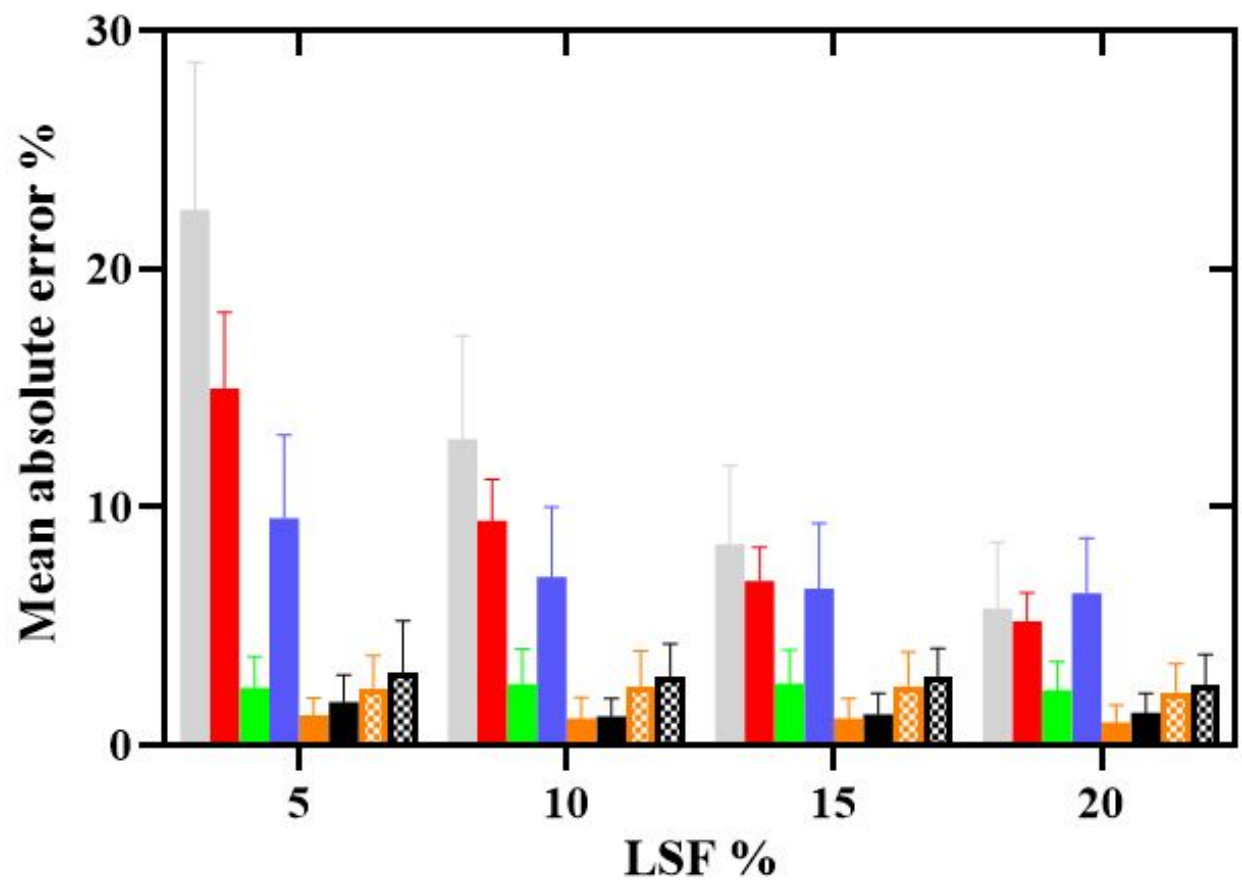

planar

SPECT/HCT-IN

SPECT/HCT-MID

SPECT/HCT-EX

SPECT/CACT

- SPECT/IACT

$\therefore$ SPECT/CACT+HCT-MID

$\approx$ SPECT/IACT+ICT-MID

Figure 12 
The mean absolute errors of LSF estimation from SPECT images with different CT protocols for AC and VOI segmentation.

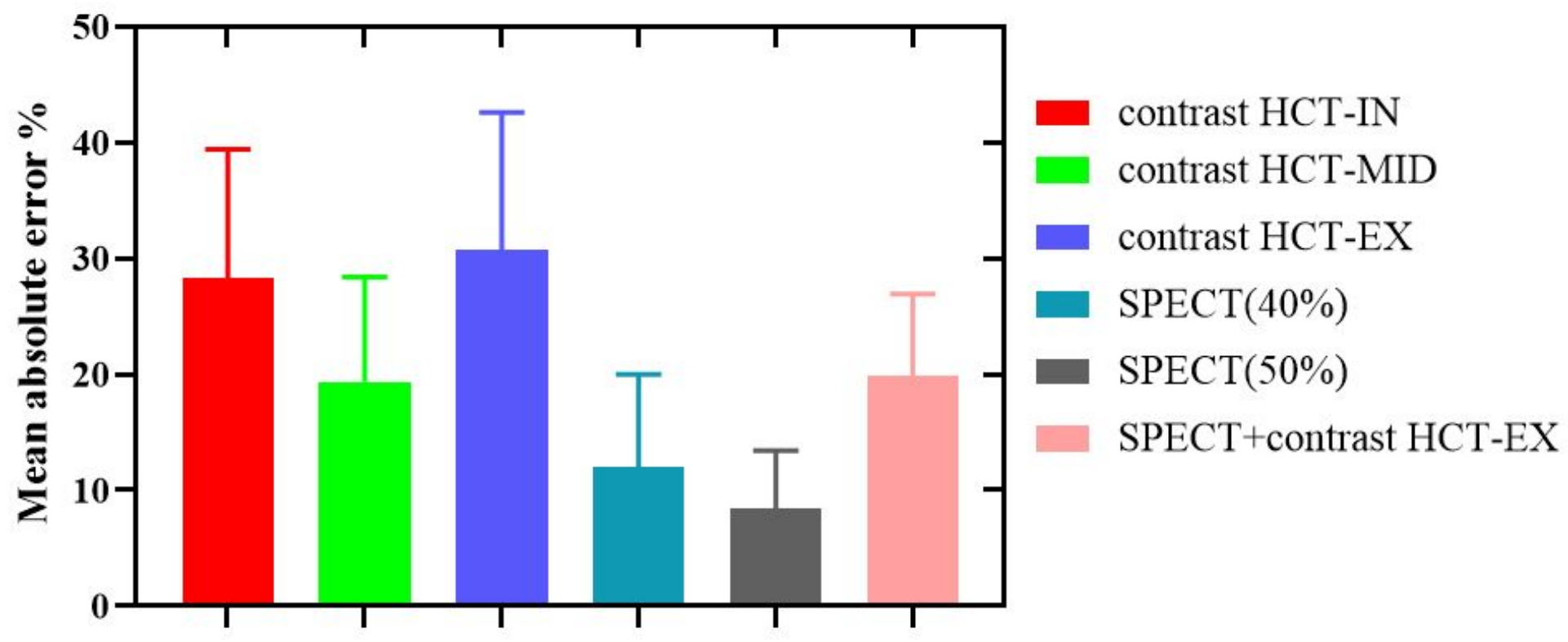

Figure 13

The mean absolute errors of TNR estimation for different tumor and NL segmentation methods for a LSF of $5 \%$.

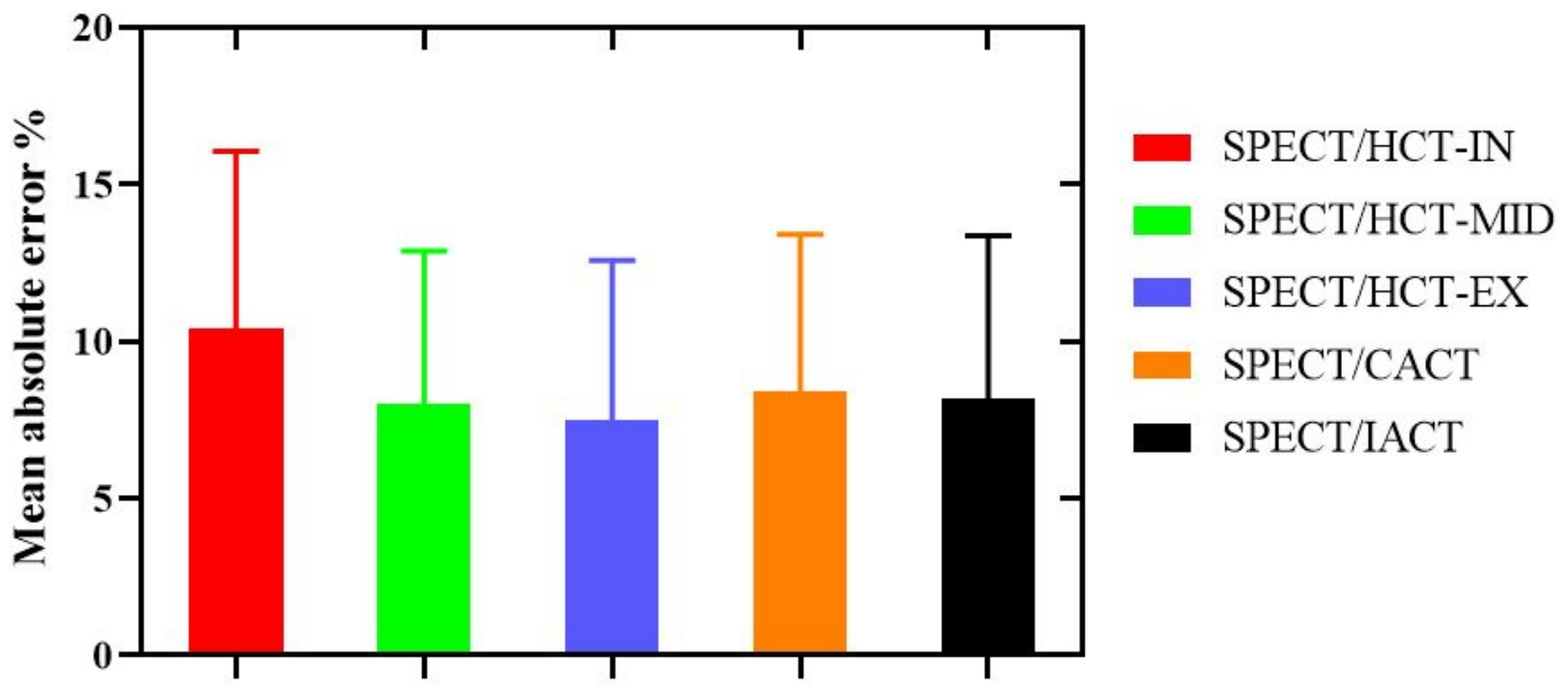

Figure 14

The mean absolute errors of TNR estimation from SPECT images with different AC protocols using SPECT $(50 \%)$ segmentation method for a LSF of $5 \%$. 


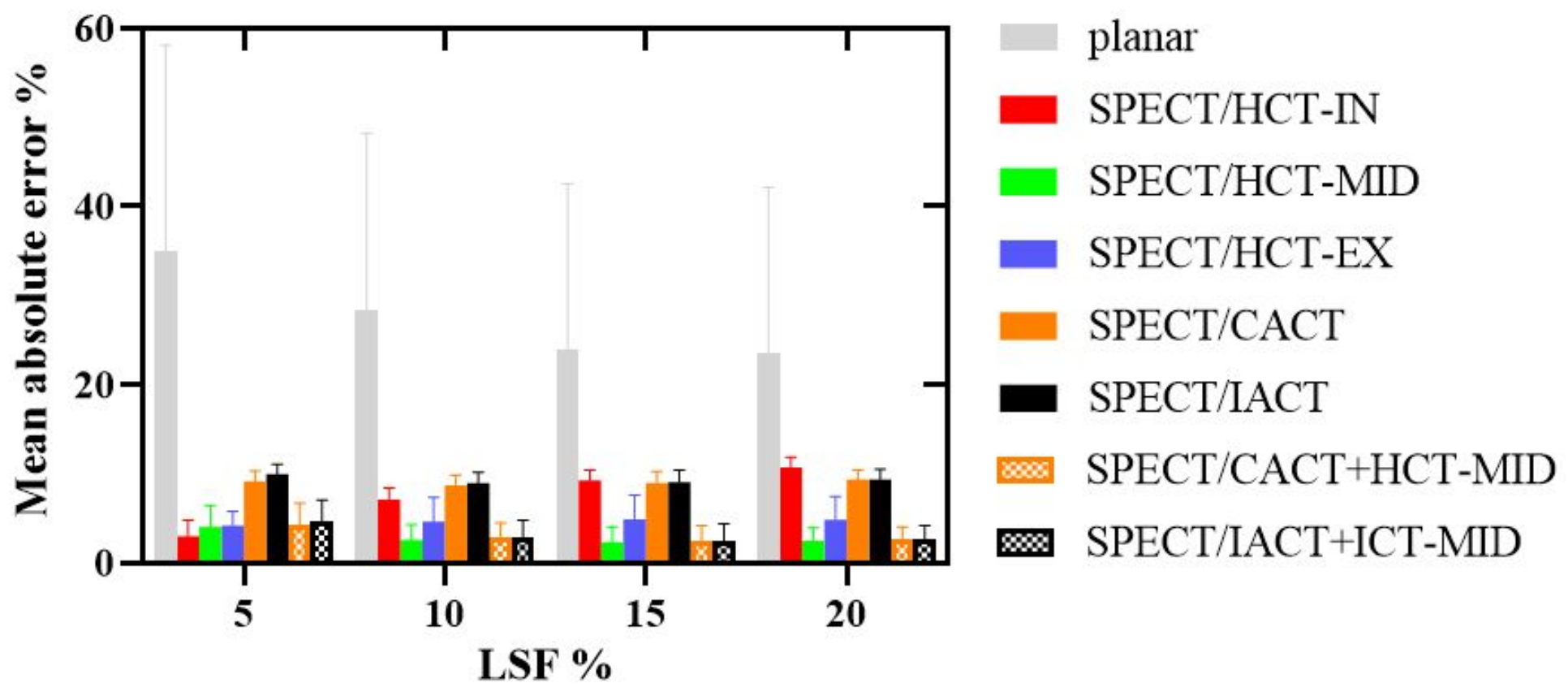

Figure 15

The mean absolute errors of lung absorbed dose estimation for 10 phantoms.

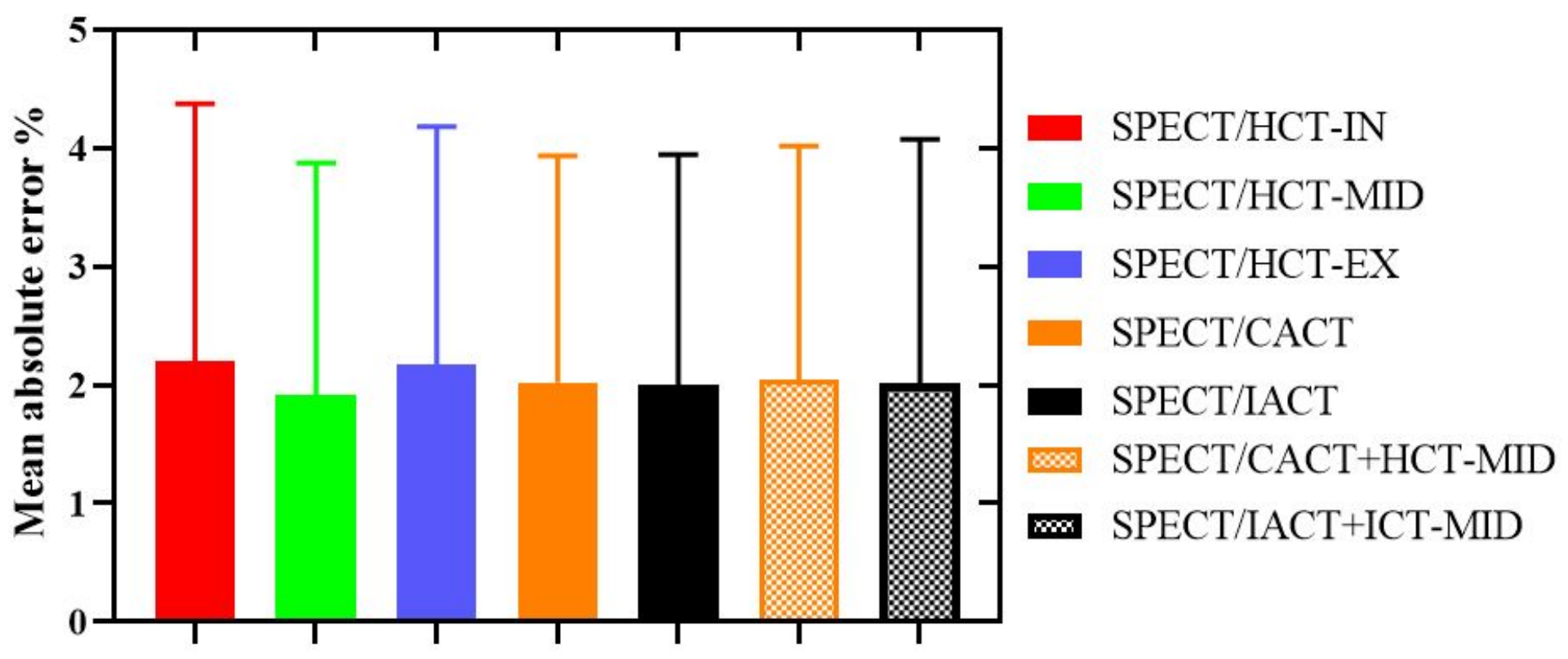

Figure 16

The mean absolute errors of NL absorbed dose estimation for 10 phantoms with LSF of $5 \%$. 


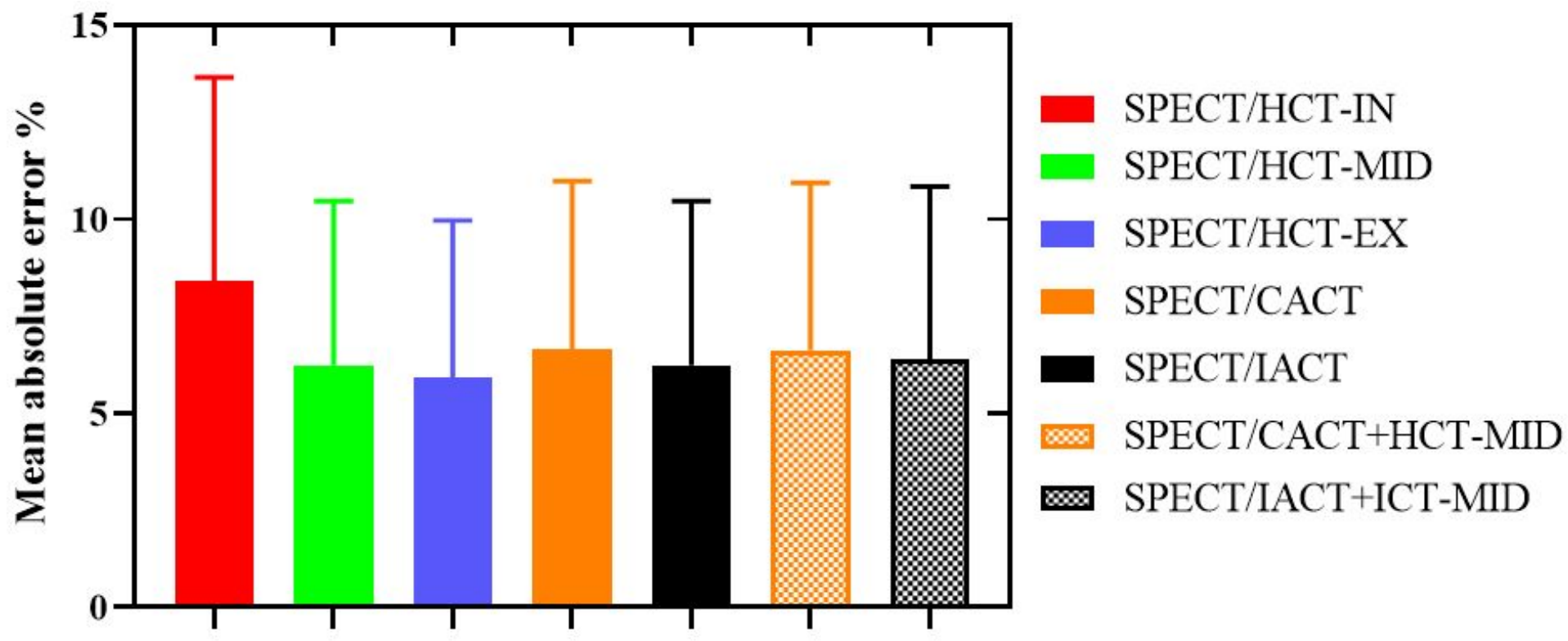

Figure 17

The mean absolute errors of tumor absorbed dose estimation for 10 phantoms with LSF of $5 \%$.

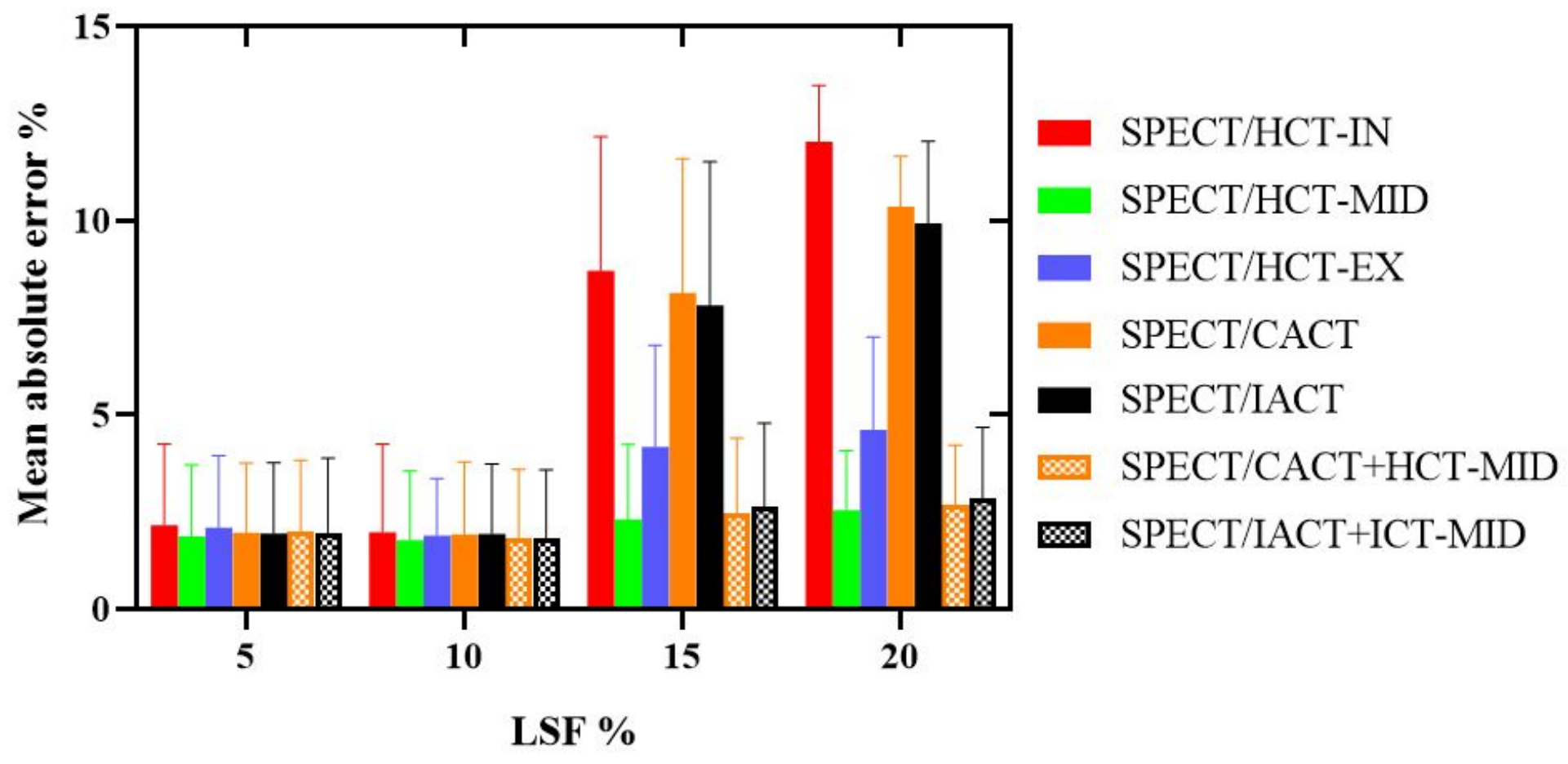

Figure 18

The mean absolute errors of IA estimation from 10 phantoms. 